Check for updates

Cite this: RSC Adv., 2017, 7, 31417

\title{
Synthesis and in vitro biological evaluation of novel diaminothiophene scaffolds as antitumor and anti- influenza virus agents. Part $2 \dagger$
}

\author{
Khurshed Bozorov, (DD ab Jiang yu Zhao, ${ }^{a}$ Li Fei Nie, ${ }^{\text {ac }}$ Hai-Rong Ma, ${ }^{a}$ \\ Khayrulla Bobakulov, ${ }^{\text {ab }}$ Rui Hu, ${ }^{\text {ac Nigora Rustamova, }}{ }^{\text {ac }}$ Guozheng Huang, (D) ${ }^{a}$ \\ Thomas Efferth ${ }^{d}$ and Haji A. Aisa (DD *a
}

\begin{abstract}
On the basis of high-throughput screening, fragment-based drug discovery, structure-activity relationships and building block analysis methods, herein we report the synthesis and biological evaluation of a novel series of diethyl 2,5-diaminothiophene-3,4-dicarboxylate derivatives. All of the prepared Schiff bases (with mono-, di- and poly-substituents at the aromatic portion), mono- and bis-amides of diethyl 2,5diaminothiophene-3,4-dicarboxylate, were evaluated against various human cancer and non-cancerous (only for active compounds) cell lines, as well as influenza A (subtypes FM/1/47/H1N1, hanfang/359/95/ H3N2) and B (subtype jifang/13/97) viruses. The obtained results suggest that some selected compounds revealed promising antitumor and antiviral activities and may serve as lead compounds for further drug discovery and development.
\end{abstract}

Received 28th April 2017

Accepted 7th June 2017

DOI: $10.1039 / c 7 r a 04808 d$

rsc.li/rsc-advances

cancerous cells, however wide chemical modification of the

\section{Introduction}

Advances in high-throughput screening (HTS), fragment-based drug discovery and building block analysis methods are powerful tools for the discovery and optimization of new drug leads in medicinal chemistry. ${ }^{1-4}$ Of particular importance are building blocks, because the drug likeness of a compound can be characterized by its building blocks. ${ }^{5}$ Likewise, this approach represents a rapid way to prepare drug candidates in medicinal chemistry. ${ }^{6}$ On the other hand, it is worth designing effective small molecules with low $\mathrm{IC}_{50}$ values at this first step of investigations. BBs containing various pharmacophore ring systems, linkers or fragments, ${ }^{7}$ play a key role for HTS-based drug discovery. ${ }^{8-10}$

Discoveries of new anticancer drug candidates are still of tremendous challenging due to the complexity of cancer biology and also of great emergency, since cancer is still the leading cause of human death in all over the world. ${ }^{11}$ Natural products remain a good arsenal for providing new entities to treat

${ }^{a}$ Key Laboratory of Plant Resources and Chemistry in Arid Regions, Xinjiang Technical Institute of Physics and Chemistry, Chinese Academy of Sciences, 40-1 South Beijing Rd, Urumqi, Xinjiang, 830011, PR China. E-mail: haji@ms.xjb.ac.cn

${ }^{b}$ Institute of the Chemistry of Plant Substances, Academy of Sciences of Uzbekistan, 77, Mirzo Ulugbek str., 100170, Tashkent, Uzbekistan

'University of Chinese Academy of Sciences, 19 A Yuquan Rd, Beijing, 100049, PR China

${ }^{d}$ Department of Pharmaceutical Biology, Institute of Pharmacy and Biochemistry, University of Mainz, 555128, Mainz, Germany

$\dagger$ Electronic supplementary information (ESI) available. See DOI: $10.1039 / \mathrm{c} 7 \mathrm{ra} 04808 \mathrm{~d}$ synthetic small molecules, as well as, their easy availability attracts more attention from pharmacists. In this way, small molecules are priority candidates for treatment of diseases in pharmacy. Among the prospectively using small molecule family in medicinal chemistry is 2-aminothiophene (2-ATs) scaffolds. Aside from their diverse biological properties, ${ }^{12-15}$ these classes of building blocks were reported as potential anticancer $^{16-18}$ and antiviral agents. ${ }^{19-21}$ Besides, cyclic 2-AT derivatives, such as thieno[2,3- $d]$ pyrimidines (TPs) also provide anticancer properties, ${ }^{22}$ as well as, act as PI3K, ${ }^{23}$ FGFR1 ${ }^{24}$ and EGFR $^{25,26}$ inhibitors, indicating many more perspectives of 2-AT libraries as initially estimated.

On the basis of these facts, we selected diethyl 2,5diaminothiophene-3,4-dicarboxylate (DDTD) building block for the present investigation containing symmetrical $\mathrm{NH}_{2}$ at $\mathrm{C}-2$ and C-5 and ester groups at C-3 and C-4 positions. Recently, we described Schiff bases of DDTD with only 4-substituents at the benzene ring (Chart 1) as potential anticancer and antimicrobial compounds and discussed the role of various substituents to DDTD under the aspect of structure activity relationships (SARs). ${ }^{27}$ Among them, some promising nitroaromatic compounds were discovered which need further modifications. On the other hand, although nitroaromatic drugs (Fig. 1) possess hepatotoxicity effects, latest reports have asserted that the ability SARs to mechanism of actions for drugs related nitro group indicates importance and perspectives to continue this area in medicinal chemistry. ${ }^{28-32}$

Thus, continuing our previous research on DDTD, we now investigated novel synthesized Schiff base (mainly were selected 


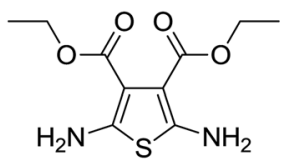

1

(DDTD)

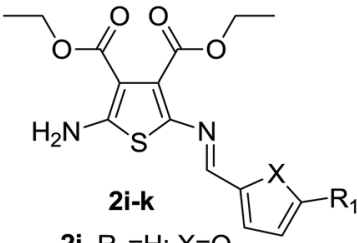

2i, $\mathrm{R}_{1}=\mathrm{H} ; \mathrm{X}=\mathrm{O}$

2j, $\mathrm{R}_{1}=\mathrm{NO}_{2} ; X=\mathrm{O}$

$\mathbf{2 k}, \mathrm{R}_{1}=\mathrm{H} ; \mathrm{X}=\mathrm{S}$

Chart 1

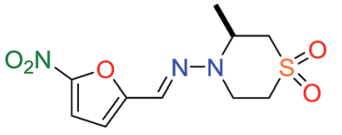

Nifurtimox

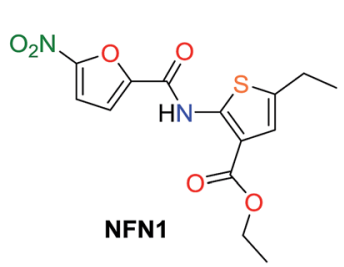

$\mathrm{O}_{2} \mathrm{~N}$<smiles>C[C@]1(COc2ccc(OC(F)(F)F)cc2)Cn2cc([N+](=O)[O-])nc2O1</smiles>

Fig. 1 Biologically relevant nitroaromatic compounds.

aldehydes with di- and poly-substituents at the aromatic portion), mono- and bis-amides of DDTD for their antiproliferative properties against cell lines of tumoral and normal (here selected most active samples) types, and influenza A and B viruses. Additionally, we also investigated the effects of compound $\mathbf{3 j}, \mathbf{3 n}$ and $\mathbf{3 p}$ on cell apoptosis compared with doxorubicin (DOX) which was used as reference drug in these class derivatives. $^{33,34}$

\section{Results and discussion}

\section{Chemistry}

The target Schiff bases (3a-p) were prepared by a previously reported synthetic route ${ }^{27,35}$ with minor changes (Scheme 1). In the present study, toluene was used as reaction solvent instead of $n$ butanol, enabling fast and clear workup procedure. The mixture of DDTD and appropriate aryl- and heteroaryl-aldehydes was
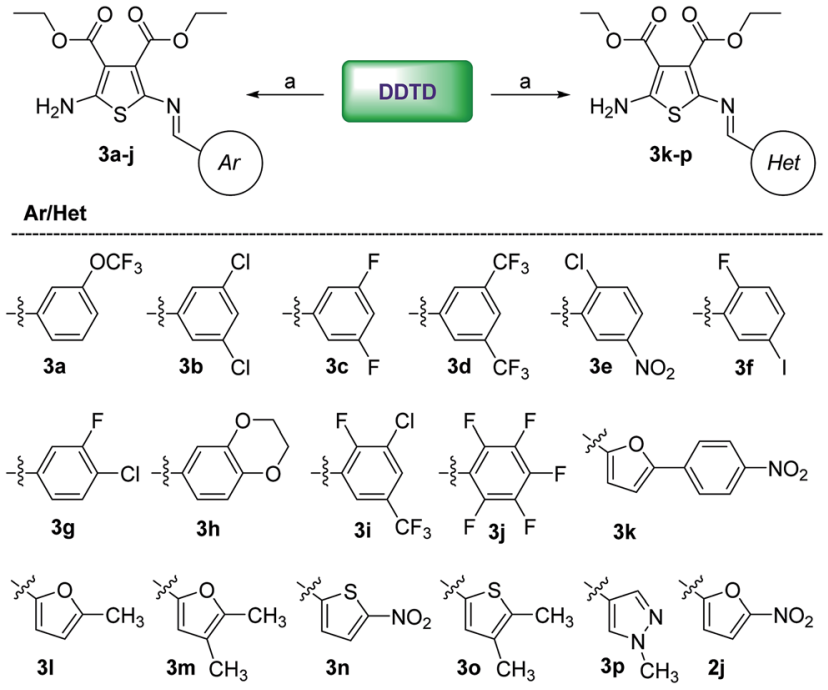

Scheme 1 Synthesis of DDTD Schiff bases 3a-p. Reagents and conditions: (a) aromatic aldehydes, toluene, reflux $30 \mathrm{~min}$.

heated at reflux in toluene for half an hour, and then evaporated in vacuo. Recrystallization of the residue from ethanol provided the target bases in excellent yield (79-94\%).

If DDTD interacted with different aromatic acid chlorides under basic conditions (methods a and b), only symmetrical bis-amides of DDTD (compounds $\mathbf{4 a - d}$ ) were obtained as final products. Particularly, this was observed with aromatic acid chlorides related to methyl group at position C-3 (4a), trifluoromethyl in positions C-3 and C-5 (4b) as well as fluoro group in positions C-3, C-4, and C-5 (4c) in the benzene portion of aromatic system. Because of planar structure of the compounds $\mathbf{4 a - d}$, it was found that by the enthalpy the optimal configuration is $Z$ forms for these compounds. Mono-amides of DDTD (5a and $\mathbf{5 c}$ ) were obtained in only two cases. It was also possible to yield mono-(5b) and symmetrical bis-(4d) amides of DDTD with 3,4,5-trimethoxybenzoyl chloride. For compound $\mathbf{5 b}$, the product yield was 53\% using method A. Applying method $\mathrm{B}$ resulted in the symmetrical bis product (4d) in excellent yield (92\%) (Scheme 2).

\section{Biological evaluation}

In vitro antiproliferative activity and SARs. All synthesized novel samples (3a-p, $\mathbf{4 a - d}$ and $\mathbf{5 a}-\mathbf{c}$ ) were evaluated for their antiproliferative activity towards four human cancer cell lines, e.g. PC-3 prostate cancer cells, A549 lung cancer cells, HCT-15 colon cancer cells and T47D breast cancer cells, which are considered as appropriate and relevant cell models in drug discovery. The inhibition concentrations required for inhibition of tumor cell proliferation of $50 \%$ ( $\mathrm{IC}_{50}$ values) of the synthesized compounds are shown in Table 1. Based on the results obtained, we drew our attention to di-substituted groups at the aromatic and heteroaromatic rings (3b-h, 3m and 3o). Likewise, we also prepared for investigation Schiff bases with diverse

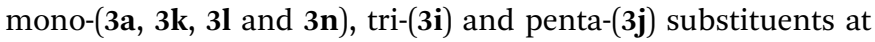
phenyl/heteryl portions, as well as the previously discovered 


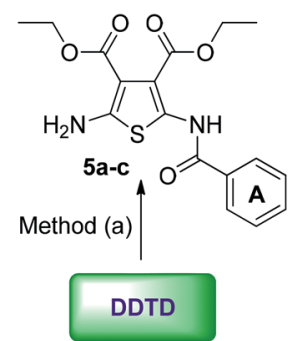

A

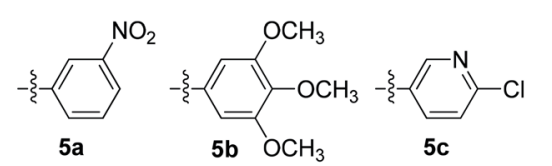

Method (b)

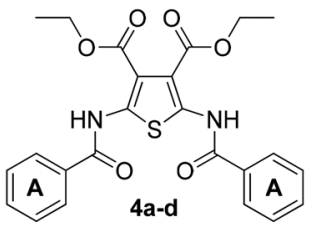

A

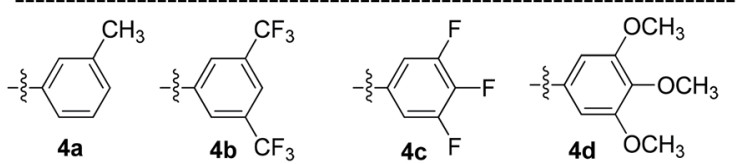

Scheme 2 Synthesis of symmetrical bis- $(4 a-d)$ and mono- $(5 a-c)$ amides of DDTD. Reagents and conditions: (method a) aromatic acid chlorides, dry benzene or chloroform, TEA, reflux 2-3 h. (Method b) aromatic acid chlorides, pyridine, r.t. 4 h.

Table 1 In vitro comparison of $\mathrm{IC}_{50}$ values human cancer cell lines of the tested compounds $3 a-p, 4 a-d$ and $5 a-c$

\begin{tabular}{|c|c|c|c|c|c|}
\hline \multicolumn{2}{|c|}{ Histotype } & \multirow{2}{*}{$\begin{array}{l}\text { Prostate } \\
\text { PC-3 }\end{array}$} & \multirow{2}{*}{$\frac{\text { Lung }}{\text { A549 }}$} & \multirow{2}{*}{$\frac{\text { Colon }}{\text { HCT-15 }}$} & \multirow{2}{*}{$\frac{\text { Breast }}{\text { T47D }}$} \\
\hline \multicolumn{2}{|c|}{ Cell lines } & & & & \\
\hline No. & Compounds & \multicolumn{4}{|c|}{$\mathrm{IC}_{50}( \pm \mathrm{SD}, \mu \mathrm{M})$} \\
\hline 1 & $2 \mathrm{j}$ & n.d. & $22.8 \pm 0.6$ & $10.2 \pm 0.6$ & $4.6 \pm 0.8$ \\
\hline 2 & $3 a$ & $33.3 \pm 0.3$ & n.d. & $34.0 \pm 0.3$ & n.d. \\
\hline 3 & $3 \mathbf{b}$ & n.d. & n.d. & n.d. & n.d. \\
\hline 4 & $3 c$ & $69.8 \pm 5.9$ & $17.9 \pm 2.1$ & $40.9 \pm 4.8$ & $20.6 \pm 2.2$ \\
\hline 5 & $3 d$ & $65.0 \pm 4.8$ & $33.0 \pm 3.5$ & $40.2 \pm 4.2$ & $51.0 \pm 5.2$ \\
\hline 6 & $3 \mathbf{e}$ & $55.0 \pm 0.5$ & n.d. & n.d. & n.d. \\
\hline 7 & $3 f$ & $45.4 \pm 4.9$ & $28.0 \pm 2.6$ & $57.7 \pm 5.8$ & $28.8 \pm 3.0$ \\
\hline 8 & $3 g$ & $42.1 \pm 5.4$ & $11.0 \pm 1.0$ & $37.3 \pm 3.6$ & $17.7 \pm 2.1$ \\
\hline 9 & $3 h$ & $29.6 \pm 0.3$ & $60.1 \pm 0.9$ & $22.3 \pm 0.4$ & $4.50 \pm 0.3$ \\
\hline 10 & $3 \mathbf{i}$ & n.d. & n.d. & n.d. & n.d. \\
\hline 11 & $3 \mathbf{j}$ & $12.0 \pm 0.7$ & $4.60 \pm 0.7$ & $2.59 \pm 0.3$ & $6.40 \pm 0.4$ \\
\hline 12 & $3 \mathbf{k}$ & $25.2 \pm 2.6$ & $28.3 \pm 2.9$ & $31.9 \pm 4.1$ & $20.6 \pm 2.3$ \\
\hline 13 & 31 & $17.3 \pm 1.8$ & $31.3 \pm 3.4$ & $25.9 \pm 2.7$ & $22.9 \pm 2.4$ \\
\hline 14 & $3 m$ & $20.9 \pm 2.6$ & $16.1 \pm 2.0$ & $30.9 \pm 3.3$ & $23.6 \pm 3.2$ \\
\hline 15 & $3 n$ & $10.9 \pm 1.3$ & $1.30 \pm 0.2$ & $6.10 \pm 0.3$ & $21.4 \pm 2.2$ \\
\hline 16 & 30 & $36.1 \pm 4.6$ & $14.8 \pm 1.5$ & $101.6 \pm 8.7$ & n.d. \\
\hline 17 & $3 \mathbf{p}$ & $15.7 \pm 1.7$ & $14.9 \pm 1.6$ & $23.3 \pm 2.5$ & $16.8 \pm 1.8$ \\
\hline 18 & $4 a$ & n.d. & $66.1 \pm 0.6$ & $12.0 \pm 0.1$ & n.d. \\
\hline 19 & $4 b$ & $11.6 \pm 0.8$ & n.d. & $12.3 \pm 0.1$ & n.d. \\
\hline 20 & $4 c$ & $9.38 \pm 0.2$ & n.d. & $14.2 \pm 0.1$ & $62.9 \pm 0.3$ \\
\hline 21 & 4d & n.d. & $71.6 \pm 0.6$ & n.d. & $12.1 \pm 0.4$ \\
\hline 22 & $5 a$ & $11.5 \pm 0.6$ & n.d. & $20.8 \pm 1.1$ & n.d. \\
\hline 23 & $5 b$ & $128.6 \pm 1.4$ & n.d. & n.d. & $6.0 \pm 0.6$ \\
\hline 24 & $5 c$ & $6.93 \pm 0.3$ & $67.0 \pm 1.1$ & $7.22 \pm 0.3$ & $22.3 \pm 0.8$ \\
\hline 25 & Doxorubicin & $7.40 \pm 1.6$ & $6.50 \pm 1.4$ & $7.92 \pm 0.3$ & $25.5 \pm 0.5$ \\
\hline
\end{tabular}

active compound $\mathbf{2 j}$. The set of heteroaromatics related Schiff bases demonstrated more significant activity results as compared to substituted phenyl related derivatives. For example, introduction of 1-methyl-1 $H$-pyrazole (3p) and 5-nitro2-thienyl (3n) fragments in most cases slowed increasing proliferation of tumoral cell lines. Interestingly, the best result was obtained with derivative $3 \mathbf{n}$, i.e. by inserting 5-nitro-2thienyl fragment in DDTD provided excellent behavior against all tumoral cell lines, which resulted high antiproliferative activity. Among the phenyl substituted Schiff base, similar behavior showed compound $\mathbf{3} \mathbf{j}$, which derived by fluorination all position of phenyl ring. Contemporaneously fluorine and chlorine substitution in aromatic ring provided significantly less toxicity dates on cancer cells (3g, towards A549 and T47D) as compared to tandem substitutions such as di-fluorine (3c), di-trifluoro methyl (3d), chlorine/nitro (3e) and fluorine/iodine (3f), while compounds with di-chlorine (3b) and 3-chloro-2-fluoro-5-(trifluoromethyl)-(3i) phenyl fragments did not show significant concentration-dependent effects. As shown in Table 1 , the majority of compounds were active against $T 47 \mathrm{D}$, in particular the compounds which contained furan/thiophene/ pyrazole linkers with various substituents (4-nitrophenyl or methyl groups) at C-4 or C-5 positions such as 3k-m and 3p. An interesting behavior was observed in case of derivatives $(3 \mathbf{c}, \mathbf{3 g}$ and 3h) which containing 3,5-difluoro-, 4-chloro-3-fluoro- or 1,4benzodioxin-substituents at the phenyl ring, these compounds showed more effective antiproliferative properties than DOX. These results confirm about many roles for fluorine substituents in medicinal chemistry, ${ }^{36,37}$ particularly in cancer chemotherapy. One of the known examples is reference drug 5-FU (5fluorouracil), ${ }^{38,39}$ which contains fluorine in the own molecule. It was observed that the majority of the selected Schiff bases showed poor antiproliferative activity $\left(\mathrm{IC}_{50} \geq 30 \mu \mathrm{M}\right)$, except of 1,4-benzodioxin-(3h), 5-methylfuran-(3l) and 1-methyl-1 $H$-pyrazole-(3p) derivatives.

In general, Table 1 shows that among the all synthesized Schiff bases, only 5-nitro-2-thienyl derivative $\mathbf{3 n}$ demonstrated antiproliferative activity on all tumoral cell lines. Perhaps, herein nitrothienyl group played a key role for increasing activity. In corresponding reports photochemical and acceptor behavior of the 5-nitro-2-thienyl group were well explained. ${ }^{\mathbf{4 0 , 4 1}}$ 
Moreover, nitrothienyl prodrugs may provide effective and selective delivery of the cytotoxic or cytostatic compound to a solid tumor. It was reported that in oxic liver homogenates, nitrothienyl related compounds was metabolically stable, as well as, when incubated in aerobic A549 whole-cell suspensions also was observe stability, which suggest that such substitution has also successfully inhibited undesired aerobic metabolism. ${ }^{\mathbf{4 2}}$ If bearing in mind that nitrothienyl containing scaffolds has been shown to demonstrate modest activity in human xenograft multiple myeloma and B-cell leukemia in vivo models, ${ }^{43}$ further developments with these class derivatives seem promising.

6-Chloronicotinamido-DDTD monoamide (5c) showed high antiproliferative activity, than DOX towards PC-3, HCT-15, and on T47D cancer cell lines, among the synthesized mono-(5a-c) and symmetrical (4a-d) amide derivatives (Table 1), which confirming anticancer drugs properties of discovered that nicotinamide containing compositions. ${ }^{44}$ Moreover, nicotinamide is a member of vitamin B family, which has no serious side effects in human organism.

Both symmetrical and mono-DDTD amides $\mathbf{4 d}$ and $\mathbf{5 b}$, which contained with 3,4,5-trimethoxy phenyl fragments, increased antiproliferative activity towards T47D cells. This finding once more confirmed the importance of the 3,4,5-trimethoxy substituent for drug discovery. ${ }^{45}$ Another symmetrical amides, which inserted substituents such as methyl at C-3 (4a), ditrifluoro methyl at C-3, C-5 (4b) and trifluoro at C-3, C-4, C-5-(4c) also resulted in considerable cytotoxicity towards HCT-15 cells.

Selectivity of $2 \mathrm{j}, 3 \mathrm{j}, 3 \mathrm{n}$ and $5 \mathrm{c}$ on normal HEK-293 cells. Compounds $\mathbf{2 j}, \mathbf{3 j}, \mathbf{3 n}$ and $\mathbf{5 c}$ which showed less $\mathrm{IC}_{50}$ values on human cancer cell lines were selected towards selectivity screening on normal human embryonic kidney (HEK-293) cells. The SI calculated by the following formula:

$$
\begin{aligned}
& \text { Selectivity index }(\mathrm{SI})= \\
& \qquad \frac{\mathrm{IC}_{50} \text { of selected samples against HEK-293 cells }}{\mathrm{IC}_{50} \text { of selected samples against cancer cells }}
\end{aligned}
$$

The results revealed that SI of these potentials was not high for compare with known anticancer drugs (Table 2). However, when SI between A549 and HEK-293 cells were calculated for derivative $3 \mathbf{n}$, satisfactory SI (50) have been obtained, which deserves further investigation. In addition, 6chloronicotinamido-DDTD monoamide (5c) demonstrated good selectivity when SI of PC-3 and HCT-25 cells were calculated between HEK-293 cells, while compound $2 \mathbf{j}$ showed similar SI value between T47D and HEK-293 cells. It was found that penta-fluoro-derivative $\mathbf{3} \mathbf{j}$ which was most potent on cancer cell lines, did not provide any selectivity.

Effects of 3j, 3n and 3p on human A549 cell cycle distribution and apoptosis induction. As shown in Table 1 , the $\mathrm{IC}_{50}$ values of compounds $\mathbf{3} \mathbf{j}$ and $\mathbf{3 n}$ were significantly lower than other DDTD derivatives. Therefore, it was interesting to study the influence of these two compounds on A549 cell growth regarding their cell cycle distribution and induction of apoptosis in comparison to DOX and compound 3p. If A549 cells were treated with various concentrations of compounds $3 \mathbf{j}$ $(0.1,0.25,0.5 \mu \mathrm{M})$ and $3 \mathrm{p}(2.5,5,15 \mu \mathrm{M})$, the cell cycle distribution did not change (Fig. 2A). However, annexin V/propidium iodide (PI) assays revealed percentages from 9.2 to $11.4 \%(3 \mathbf{j})$ and 4.1 to $9.6 \%(3 \mathrm{p})\left(Q_{2}+Q_{4}\right)$ at different concentrations $(p<$ 0.01 ) (Fig. 2B and C). The apoptosis rates of DOX ranged from 10.3 to $16.3 \%\left(Q_{2}+Q_{4}\right)(p<0.01)$. In addition, if A549 cells were treated with compound $3 \mathbf{n}$ at low micromolar concentrations $(0.1,0.25,0.5 \mu \mathrm{M})$, and the induction of apoptosis increased from 8.4 to $12.6 \%\left(Q_{2}+Q_{4}\right)$ (Fig. 2B, C).

Anti-influenza activity. The target compounds (2j, 3a-p, 4ad and $\mathbf{5 a}-\mathbf{c}$ ) were screened against influenza $\mathrm{A}$ virus of the subtypes FM/1/47/H1N1, hanfang/359/95/H3N2 and influenza B virus of the subtype jifang/13/97 (CPE) using MDCK cells with oseltamivir and ribavirin (RBV) as positive controls. In our initial investigation, several thiophene-3,5-dicarboxalate derivatives $(\mathbf{2} \mathbf{j}, \mathbf{3 a}, \mathbf{3} \mathbf{g}, \mathbf{3} \mathbf{j}, \mathbf{3 k}, \mathbf{3 n}-\mathbf{p}$ and $\mathbf{5 a})$ displayed against the $\mathrm{H} 1 \mathrm{~N} 1$ subtype potent activity with $\mathrm{IC}_{50}$ values ranging from 0.94 to $10.69 \mu \mathrm{M}$. In particular compound $2 \mathrm{j}\left(\mathrm{IC}_{50}=0.94 \mu \mathrm{M}\right)$ was more active than oseltamivir and RBV (Table 3). Similar results were observed regarding influenza subtype H3N2 and compounds 2j, 3a, 3b, 3d, 3g, 3h, 3k, 3l, 3n and 3p. Especially, 4-chloro-3-fluorophenyl related Schiff base $\left(3 \mathrm{~g}, \mathrm{IC}_{50}=1.81\right)$ showed similar anti-H3N2 activity as the positive control drugs. SARs of selected compounds regarding inhibition of influenza A and B viruses suggest that introduction of diverse pharmacophore substituents, such as nitro-furfuryl and fluorine significantly increased inhibition activity. For instance, derivative $\mathbf{2 j}$ with a 5-nitro-furanyl substituent showed the best antiinfluenza activity against A and B subtypes. 4-Chloro-3fluorophenyl-related compound $\mathbf{3 g}$ also showed high activity against influenza H3N2 virus. Among the mono- and bis amides of DDTD, only mono amide 5a with a 3-nitrophenyl group demonstrated good anti-influenza activity. However, bis amides of DDTD have demonstrated inactive on influenza A and B viruses. Although, several compounds showed potential antiviral activity, the most active compounds did not demonstrate high selectivity indexes (SI) and 50\% cytotoxic concentration

\begin{tabular}{|c|c|c|c|c|c|}
\hline Compounds & $\mathrm{IC}_{50}( \pm \mathrm{SD}, \mu \mathrm{M})$ & $\mathrm{SI}^{\mathrm{PC}-3}$ & $\mathrm{SI}^{\mathrm{A} 549}$ & $\mathrm{SI}^{\mathrm{HCT}-15}$ & $\mathrm{SI}^{\mathrm{T} 47 \mathrm{D}}$ \\
\hline $2 j$ & $111.3 \pm 2.8$ & nd & $4.9 \pm 4.7$ & $11 \pm 4.7$ & $24.1 \pm 3.5$ \\
\hline $3 \mathbf{j}$ & $31.4 \pm 1.7$ & $2.6 \pm 2.4$ & $6.8 \pm 2.4$ & $12.1 \pm 5.6$ & $4.9 \pm 4.2$ \\
\hline $5 c$ & $160.0 \pm 2.3$ & $23 \pm 7.7$ & $2.4 \pm 2.0$ & $22 \pm 7.7$ & $7.1 \pm 2.9$ \\
\hline
\end{tabular}

Table $2 \quad I_{50}$ values on normal human embryonic kidney (HEK-293) cell and SI values of antiproliferative compounds 

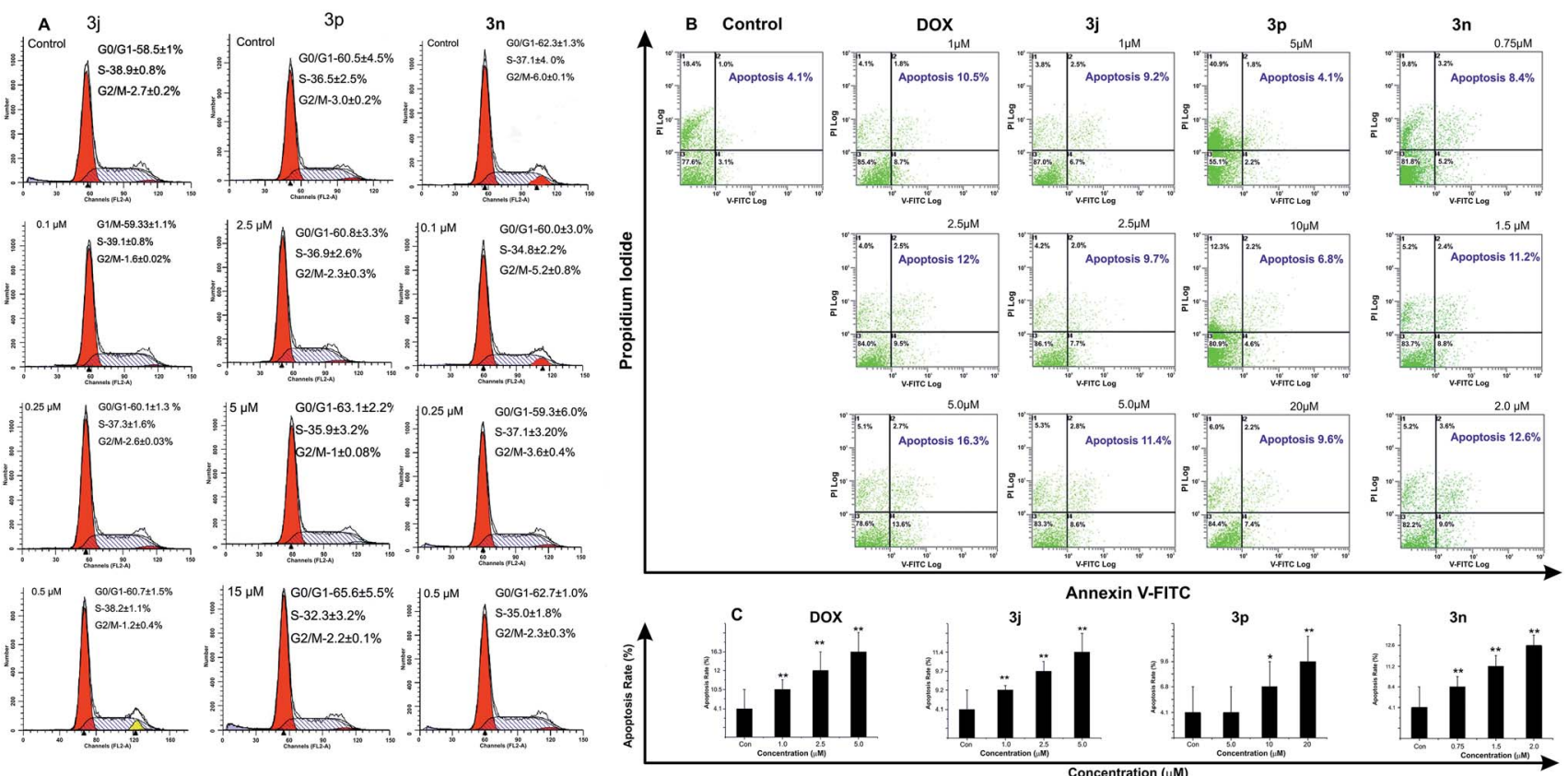

Fig. 2 (A) Effects of compounds 3j, 3p and $3 n$ on cell cycle distribution in A549 cells. (B) The induction of apoptosis in A549 cells of DOX, 3j, 3p and $3 \mathrm{n}$ stained with PI and Annexin V-FITC after treatment at different concentrations for $12 \mathrm{~h} . \mathrm{Q}_{1}[\mathrm{FITC}(-) / \mathrm{PI}(+)]$, necrotic cells; $Q_{2}[\mathrm{FITC}(+) /$ $\mathrm{PI}(+)$ ], late apoptotic cells; $Q_{3}[$ Annexin $\mathrm{V}(+) / \mathrm{PI}(+)]$, living cells; $Q_{4}[\mathrm{FITC}(+) / \mathrm{PI}(-)]$, early apoptotic cells. (C) Percentage of apoptotic A549 cells after treatment with samples DOX, 3j, 3p and $3 n$ at different concentrations.

Table 3 Inhibitory activity of the compound $2 \mathrm{j}$ and novel compounds $3 a-p, 4 a-d$ and $5 a-c$ against influenza $A$ and $B$ viruses

\begin{tabular}{|c|c|c|c|c|c|c|c|}
\hline \multirow[b]{2}{*}{ Compounds } & \multirow[b]{2}{*}{$\mathrm{TC}_{50}{ }^{a}(\mu \mathrm{M})$} & \multicolumn{2}{|c|}{ Anti-influenza H1N1 virus } & \multicolumn{2}{|c|}{ Anti-influenza H3N2 virus } & \multicolumn{2}{|c|}{ Anti-influenza B virus } \\
\hline & & $\mathrm{IC}_{50}{ }^{b}$ & $\mathrm{SI}^{c}$ & $\mathrm{IC}_{50}$ & SI & $\mathrm{IC}_{50}$ & SI \\
\hline $3 \mathbf{a}$ & 10.69 & $6.28 \pm 0.08$ & 1.7 & $2.07 \pm 0.91$ & 5.7 & $9.54 \pm 0.90$ & 1.1 \\
\hline $3 \mathbf{b}$ & 32.08 & $>166.67$ & $>3.0$ & $11.40 \pm 1.19$ & 2.8 & $7.37 \pm 1.10$ & 4.3 \\
\hline $3 \mathrm{c}$ & $>500$ & $>166.67$ & $\mathrm{nd}^{e}$ & $111.12 \pm 78.57$ & 6.0 & $131.45 \pm 49.81$ & 4.1 \\
\hline $3 f$ & 129.34 & $21.32 \pm 3.21$ & 6.0 & $24.57 \pm 3.15$ & 5.3 & $42.17 \pm 6.35$ & 3.1 \\
\hline $3 \mathbf{g}$ & 73.12 & $7.41 \pm 1.94$ & 9.9 & $1.81 \pm 0.35$ & 41.2 & $18.26 \pm 3.10$ & 4.0 \\
\hline $3 \mathbf{h}$ & 129.34 & $18.75 \pm 2.45$ & 6.9 & $11.25 \pm 4.42$ & 12.5 & $39.17 \pm 8.90$ & 3.3 \\
\hline $3 \mathbf{i}$ & $>500$ & $>166.67$ & $>3.0$ & $>166.67$ & $>3.0$ & $>166.67$ & $>3.0$ \\
\hline $3 \mathbf{j}$ & 32.08 & $10.69 \pm 0.01$ & 3.0 & $26.73 \pm 3.73$ & 1.2 & $14.40 \pm 0.04$ & 2.2 \\
\hline $3 \mathbf{k}$ & 38.52 & $10.69 \pm 0.06$ & 3.6 & $2.63 \pm 0.01$ & 14.6 & $14.30 \pm 0.01$ & 2.7 \\
\hline $3 p$ & 10.69 & $4.54 \pm 0.36$ & 2.2 & $9.81 \pm 1.31$ & 1.1 & $8.29 \pm 0.90$ & 1.3 \\
\hline $4 a$ & 166.67 & $37.60 \pm 7.81$ & 4.6 & $79.60 \pm 8.62$ & 2.1 & $55.52 \pm 5.75$ & 2.9 \\
\hline $4 b$ & 240.37 & $52.50 \pm 9.54$ & 4.6 & $67.34 \pm 7.45$ & 3.6 & $59.11 \pm 3.50$ & 4.0 \\
\hline $4 \mathrm{c}$ & 288.68 & $145.18 \pm 21.90$ & 2.0 & $>166.67$ & $>3.0$ & $>166.67$ & nd \\
\hline $4 d$ & 166.67 & $32.08 \pm 0.02$ & 5.2 & $62.26 \pm 5.66$ & 2.7 & $55.60 \pm 4.63$ & 3.0 \\
\hline $5 \mathbf{a}$ & 240.37 & $18.52 \pm 0.05$ & 13 & $>166.67$ & $>3.0$ & $9.32 \pm 1.94$ & 10.6 \\
\hline $5 \mathbf{b}$ & 240.37 & $32.08 \pm 0.01$ & 7.5 & $49.34 \pm 8.80$ & 5.0 & $43.12 \pm 0.03$ & 5.6 \\
\hline $5 c$ & 346.68 & $42.21 \pm 0.02$ & 8.2 & $56.90 \pm 20.77$ & 6.4 & $100.47 \pm 40.84$ & 3.8 \\
\hline Oseltamivir & 1260 & $3.56 \pm 0.53$ & 343.4 & $1.39 \pm 0.37$ & 915.3 & $119.58 \pm 5.81$ & 10.6 \\
\hline RBV & 1164.1 & $2.62 \pm 0.79$ & 465.6 & $1.77 \pm 1.00$ & 784.8 & $1.13 \pm 0.43$ & 1116.9 \\
\hline
\end{tabular}

${ }^{a} 50 \%$ cytotoxic concentration. ${ }^{b} 50 \%$ virus-inhibitory concentration, determined by the $\mathrm{CPE}$ inhibition assay. ${ }^{c}$ Selectivity index $\left(\mathrm{TC}_{50} / \mathrm{IC}_{50}\right)$.

${ }^{d}$ Results are expressed as mean values $\left( \pm\right.$ SEM) derived from two replicates. ${ }^{e}$ Not determined. 
$\left(\mathrm{TC}_{50}\right)$ except of compound $3 \mathrm{~g}\left(\mathrm{SI}=41.2\right.$ on $\mathrm{H} 3 \mathrm{~N} 2$ virus, $\mathrm{TC}_{50}=$ 73.12). We thus investigated influenza inhibition of DDTD derivatives and our obtained results suggest that further structural modification of these scaffolds against influenza viruses may be promising. As confirm our assumption, recently it was reported that, if cycloheptathiophene-3-carboxamide scaffold, ${ }^{20}$ when properly functionalized, emerged as particularly suitable to impart anti-Flu activity and these derivatives effectively inhibited all FluA strains tested, of both H1N1 and H3N2 subtypes, as well as, were suggested its peculiar mechanism of action. $^{20}$

\section{Conclusions}

Recently, we had discovered Schiff bases of DDTD (with only 4-substituents at the benzene ring) as potential anticancer and antimicrobial agents, ${ }^{27}$ now in this investigation we have developed our previous work with additional assays, such as anti-influenza virus activities, cell cycle and apoptotic analysis, also have been calculated SI of promising compounds by using human normal cell (HEK-293) as well as have synthesized novel mono- and bis-amides and Schiff bases (in this time were selected aldehydes with di- and poly-substituents at the aromatic portion) of DDTD on the basis SARs. It was observed that, DDTD derivatives $(\mathbf{3} \mathbf{j}, \mathbf{3 n}, \mathbf{3 p}$, and $\mathbf{5 c})$ showed significant cytotoxicity against different human tumor cells. In particular, 2-amino-5-[(E)-[(5nitrothiophen-2-yl)methylidene]amino]thiophene-derivative (3n) showed strong cytotoxic activity and selectivity. It significantly induced apoptosis in A549 cells at low micromolar concentrations. Regarding DDTD amides, 2-(6-chloronicotinamido-) containing amide $\mathbf{5 c}$ displayed consistent antiproliferative activity showing lower $\mathrm{IC}_{50}$ values than DOX in PC-3 prostate, HCT-15 colon and T47D breast cancer cell lines. Moreover, evaluation of target compounds against influenza A and B viruses showed moderate activity, in particular potential hit compound $2 \mathbf{j}$ and $3 \mathbf{g}$ were more or similar active than or with control drugs oseltamivir and RBV. Thus, we concluded that our results are promising for the future design of antitumor and antiviral activity of DDTD derivatives.

\section{Experimental section}

\section{Chemistry}

Materials and methods. The NMR spectra were recorded on a Varian 400-MR and Varian VNMRS-600 spectrometer (400 $\mathrm{MHz}$ and $600 \mathrm{MHz}$ for ${ }^{1} \mathrm{H}$ ) using $\mathrm{CDCl}_{3}$ as a solvent and TMS as an internal standard in ${ }^{1} \mathrm{H}$ NMR spectra. In ${ }^{13} \mathrm{C}$ NMR spectra chemical shifts were internally referenced to the solvent signals $\left(\mathrm{CDCl}_{3} 77.16 \mathrm{ppm}\right.$ related to TMS). Mass spectra were acquired on QSTAR Elite LC-MS/MS system (Applied Biosystems/MDS Sciex Concord, ON Canada) spectrometers. Melting points were determined using a BUCHI Melting Point B-540 apparatus (Germany) and were uncorrected. All reagents (aromatic aldehydes and acid chlorides) and solvents were purchased from Tokyo Chemical Industry Co., Ltd. (Tokyo, Japan), Energy Chemical Technology Co., Ltd., and Shanghai Qinghang Chemical Co., Ltd. (Shanghai, China) and were used without further purification. Purities of the target compounds were established by analytical HPLC: Dionex Ultimate 3000 HPLC system with DAD detection at $254 \mathrm{~nm}$. The chromatography was performed on Sunfire C18 column from Waters, USA, having the following specifications: internal diameter $4.6 \mathrm{~mm}$, height $250 \mathrm{~mm}$, and particle size $5 \mu \mathrm{m}$. The method used had a flow of

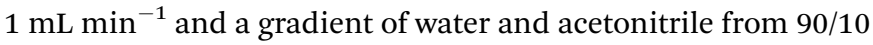
to $0 / 100$. All tested compounds are $>95 \%$ pure.

\section{General procedure for the synthesis of DDTD (1)}

A mixture of elemental sulfur $10 \mathrm{~g}$ (312 mmol), ethylcyanoacetate (71.2 g; $623 \mathrm{mmol})$, DMF $(50 \mathrm{~mL})$ and TEA $(10 \mathrm{~mL}$; $71 \mathrm{mmol}$ ) was allowed to react for 6 days with continuous stirring at $15{ }^{\circ} \mathrm{C}$. The obtained mixture was added to $3 \mathrm{~L}$ cool water and stirred over $4 \mathrm{~h}$. The precipitate was filtered and dried giving a yellow solid (59 g; 73\%).

\section{General procedure for the synthesis of compounds $3 a-p$}

Aromatic aldehydes $(0.3 \mathrm{mmol})$ were added to a $0.2 \mathrm{mmol}$ solution of the DDTD (1) in toluene $(10 \mathrm{~mL})$. The mixture was refluxed for $0.5 \mathrm{~h}$. Further, the solution was evaporated at low pressure and the residue was purified and recrystallized from ethanol.

Diethyl 2-amino-5-[(E)-[(3-trifluoromethoxyphenyl)methylidene] amino]thiophene-3,4-dicarboxylate (3a). Yield: $82 \%$; yellow solid, mp $127-129{ }^{\circ} \mathrm{C}$. HPLC purity $98.4 \%\left(t_{\mathrm{R}}=\right.$ $25.86 \mathrm{~min}) .{ }^{1} \mathrm{H} \mathrm{NMR}\left(\mathrm{CDCl}_{3}, 400 \mathrm{MHz}, \delta \mathrm{ppm}\right): 7.93$ (s, 1H, $=$ $\mathrm{CH}$ ), 7.62 (m, 2H, Ar-H), 7.41 (t, J=7.9 Hz, 1H, Ar-H), 7.23 (br.d, $J=8.0 \mathrm{~Hz}, 1 \mathrm{H}, \mathrm{Ar}-\mathrm{H}$ ), 6.41 (br.s, $2 \mathrm{H}, \mathrm{NH}_{2}$ ), 4.43 (q, $J=7.1 \mathrm{~Hz}$, $\left.2 \mathrm{H}, \mathrm{OCH}_{2} \mathrm{CH}_{3}\right), 4.26$ (q, $\left.J=7.1 \mathrm{~Hz}, 2 \mathrm{H}, \mathrm{OCH}_{2} \mathrm{CH}_{3}\right), 1.44(\mathrm{t}, J=$ $\left.7.1 \mathrm{~Hz}, 3 \mathrm{H}, \mathrm{OCH}_{2} \boldsymbol{C H}_{3}\right), 1.32\left(\mathrm{t}, J=7.1 \mathrm{~Hz}, 3 \mathrm{H}, \mathrm{OCH}_{2} \boldsymbol{C H}_{3}\right) \cdot{ }^{13} \mathrm{C}$ NMR ( $\left.\mathrm{CDCl}_{3}, 100 \mathrm{MHz}, \delta \mathrm{ppm}\right): 165.46(\mathrm{C}=\mathrm{O}), 164.49(\mathrm{C}=\mathrm{O})$, $160.26\left(\mathrm{NH}_{2} \mathrm{CS}\right), 150.62(\mathrm{~N}=\mathrm{CH}), 149.79$ (Ar-C), 138.04 (Ar-C), 133.43 (C), 131.28 (C), 130.18 (Ar-CH), 127.00 (Ar-CH), 123.09 (Ar-CH), 120.59 (q, $\left.J=257.7 \mathrm{~Hz}, \mathrm{CF}_{3}\right), 119.86(\mathrm{Ar}-\mathrm{CH}), 103.34$ (C), $61.78\left(\mathrm{CH}_{2}\right), 60.52\left(\mathrm{CH}_{2}\right), 14.45\left(\mathrm{CH}_{3}\right), 14.31\left(\mathrm{CH}_{3}\right)$. MS (ESI): $\mathrm{m} / \mathrm{z} 431[\mathrm{M}+\mathrm{H}]^{+}$.

Diethyl 2-amino-5-[(E)-[(3,5-dichlorophenyl)methylidene]amino]thiophene-3,4-dicarboxylate (3b). Yield: $86 \%$; yellow solid, mp $223-225{ }^{\circ} \mathrm{C}$. HPLC purity $97.9 \%\left(t_{\mathrm{R}}=27.97 \mathrm{~min}\right) .{ }^{1} \mathrm{H}$ $\operatorname{NMR}\left(\mathrm{CDCl}_{3}, 400 \mathrm{MHz}, \delta \mathrm{ppm}\right): 7.80(\mathrm{~s}, 1 \mathrm{H}, \mathrm{N}=\mathrm{CH}), 7.58(\mathrm{~d}, J=$ $1.9 \mathrm{~Hz}, 2 \mathrm{H}, \mathrm{Ar}-\mathrm{H}$ ), 7.35 (t, $J=1.9 \mathrm{~Hz}, 1 \mathrm{H}, \mathrm{Ar}-\mathrm{H}$ ), 6.44 (br.s, $2 \mathrm{H}$, $\left.\mathrm{NH}_{2}\right), 4.44\left(\mathrm{q}, J=7.1 \mathrm{~Hz}, 2 \mathrm{H}, \mathrm{OCH}_{2} \mathrm{CH}_{3}\right), 4.26(\mathrm{q}, J=7.1 \mathrm{~Hz}, 2 \mathrm{H}$, $\left.\mathrm{OCH}_{2} \mathrm{CH}_{3}\right), 1.45\left(\mathrm{t}, J=7.1 \mathrm{~Hz}, 3 \mathrm{H}, \mathrm{OCH}_{2} \boldsymbol{C H}_{3}\right), 1.32(\mathrm{t}, J=7.1 \mathrm{~Hz}$, $\left.3 \mathrm{H}, \mathrm{OCH}_{2} \boldsymbol{C H}_{3}\right) \cdot{ }^{13} \mathrm{C} \mathrm{NMR}\left(\mathrm{CDCl}_{3}, 100 \mathrm{MHz}, \delta \mathrm{ppm}\right): 165.34(\mathrm{C}=$ $\mathrm{O}), 164.41(\mathrm{C}=\mathrm{O}), 160.52\left(\mathrm{NH}_{2} C \mathrm{~S}\right), 149.04(\mathrm{~N}=C \mathrm{H}), 138.77(\mathrm{C})$, 135.50 (2Ar-C), 132.99 (Ar-C), 131.94 (C), 130.35 (Ar-CH), 126.46 (2Ar-CH), $103.43(\mathrm{C}), 61.86\left(\mathrm{CH}_{2}\right), 60.57\left(\mathrm{CH}_{2}\right), 14.50\left(\mathrm{CH}_{3}\right)$, $14.32\left(\mathrm{CH}_{3}\right)$. MS (ESI): $m / z 416[\mathrm{M}+\mathrm{H}]^{+}$.

Diethyl 2-amino-5-[(E)-[(3,5-difluorophenyl)methylidene]amino]thiophene-3,4-dicarboxylate (3c). Yield: 89\%; yellow solid, mp $182-184{ }^{\circ} \mathrm{C}$. HPLC purity $100 \%\left(t_{\mathrm{R}}=24.08 \mathrm{~min}\right) .{ }^{1} \mathrm{H}$ $\mathrm{NMR}\left(\mathrm{CDCl}_{3}, 400 \mathrm{MHz}, \delta \mathrm{ppm}\right): 8.13(\mathrm{~s}, 1 \mathrm{H}, \mathrm{N}=\mathrm{CH}), 7.98(\mathrm{td}, J=$ 8.5 ; $6.6 \mathrm{~Hz}, 1 \mathrm{H}, \mathrm{Ar}-\mathrm{H}), 6.91$ (td, $J=8.5,2.5 \mathrm{~Hz}, 1 \mathrm{H}, \mathrm{Ar}-\mathrm{H}), 6.81$ (ddd, $J=11.0,8.5,2.5 \mathrm{~Hz}, 1 \mathrm{H}, \mathrm{Ar}-\mathrm{H}$ ), 6.50 (br.s, $2 \mathrm{H}, \mathrm{NH}_{2}$ ), 4.41 (q, $\left.J=7.1 \mathrm{~Hz}, 2 \mathrm{H}, \mathrm{OCH}_{2} \mathrm{CH}_{3}\right), 4.25\left(\mathrm{q}, J=7.1 \mathrm{~Hz}, 2 \mathrm{H}, \mathrm{OCH}_{2} \mathrm{CH}_{3}\right), 1.42$ 
(t, $\left.J=7.1 \mathrm{~Hz}, 3 \mathrm{H}, \mathrm{OCH}_{2} \boldsymbol{C H}_{3}\right), 1.31\left(\mathrm{t}, J=7.1 \mathrm{~Hz}, 3 \mathrm{H}, \mathrm{OCH}_{2} \boldsymbol{C H}_{3}\right)$. ${ }^{13} \mathrm{C} \mathrm{NMR}\left(\mathrm{CDCl}_{3}, 100 \mathrm{MHz}, \delta \mathrm{ppm}\right): 165.66(\mathrm{C}=\mathrm{O}), 164.60(\mathrm{dd}, J=$ 254.5, $12.2 \mathrm{~Hz}, \mathrm{Ar}-\mathrm{CF}$ ), 164.44 (C=O), 162.50 (dd, $J=256.0$, $11.9 \mathrm{~Hz}, \mathrm{Ar}-\mathrm{CF}$ ), $160.36\left(\mathrm{NH}_{2} \mathrm{CS}\right), 144.40$ (dd, $J=4.6,2.3 \mathrm{~Hz}, \mathrm{~N}=$ CH), 134.11 (C), 130.69 (C), 129.12 (dd, $J=9.8,4.2 \mathrm{~Hz}, \mathrm{Ar}-\mathrm{C}$ ), 120.39 (dd, $J=9.4,3.9 \mathrm{~Hz}$, Ar-C), 112.38 (dd, $J=21.9,3.5 \mathrm{~Hz}$, Ar-C), 104.08 (t, $J=25.31 \mathrm{~Hz}, \mathrm{Ar}-\mathrm{C}), 103.00(\mathrm{C}), 61.70\left(\mathrm{CH}_{2}\right), 60.41$ $\left(\mathrm{CH}_{2}\right), 14.43\left(\mathrm{CH}_{3}\right), 14.31\left(\mathrm{CH}_{3}\right)$. MS (ESI): $m / z 383[\mathrm{M}+\mathrm{H}]^{+}$.

Diethyl 2-amino-5-[(E)-[(3,5-bis-trifluoromethylphenyl)methylidene]amino]thiophene-3,4-dicarboxylate (3d). Yield: $85 \%$; yellow solid, mp 228-230 ${ }^{\circ} \mathrm{C}$. HPLC purity $99.1 \%\left(t_{\mathrm{R}}=\right.$ $27.99 \mathrm{~min}) .{ }^{1} \mathrm{H}$ NMR $\left(\mathrm{CDCl}_{3}, 400 \mathrm{MHz}, \delta \mathrm{ppm}\right): 8.16$ (s, 2H, Ar$\mathrm{H}), 7.99(\mathrm{~s}, 1 \mathrm{H}, \mathrm{N}=\mathrm{CH}), 7.86$ (s, 1H, Ar-H), 6.45 (br.s, 2H, $\left.\mathrm{NH}_{2}\right), 4.45\left(\mathrm{q}, J=7.1 \mathrm{~Hz}, 2 \mathrm{H}, \mathrm{OCH}_{2} \mathrm{CH}_{3}\right), 4.27(\mathrm{q}, J=7.1 \mathrm{~Hz}, 2 \mathrm{H}$, $\left.\mathrm{OCH}_{2} \mathrm{CH}_{3}\right), 1.46\left(\mathrm{t}, J=7.1 \mathrm{~Hz}, 3 \mathrm{H}, \mathrm{OCH}_{2} \boldsymbol{C H}_{3}\right), 1.32(\mathrm{t}, J=7.1 \mathrm{~Hz}$, $\left.3 \mathrm{H}, \mathrm{OCH}_{2} \boldsymbol{C H}_{3}\right) \cdot{ }^{13} \mathrm{C} \mathrm{NMR}\left(\mathrm{CDCl}_{3}, 100 \mathrm{MHz}, \delta \mathrm{ppm}\right): 165.20(\mathrm{C}=$ O), $164.38(\mathrm{C}=\mathrm{O}), 160.77\left(\mathrm{NH}_{2} C \mathrm{~S}\right), 148.07(\mathrm{~N}=C \mathrm{H}), 137.92(\mathrm{Ar}-$ C), 132.97 (C), 132.43 (C), 132.16 (q, $J=32.4 \mathrm{~Hz}, 2 \mathrm{Ar}-\mathrm{C}), 127.87$ (2Ar-CH), 123.65 (Ar-CH), 123.23 (q, $J=272.8 \mathrm{~Hz}, 2 \mathrm{CF}_{3}$ ), 103.61 (C), $61.93\left(\mathrm{CH}_{2}\right), 60.67\left(\mathrm{CH}_{2}\right), 14.45\left(\mathrm{CH}_{3}\right), 14.28\left(\mathrm{CH}_{3}\right) . \mathrm{MS}$ (ESI): $m / z 483[\mathrm{M}+\mathrm{H}]^{+}$.

Diethyl 2-amino-5-[(E)-[(2-chloro-5-nitrophenyl)methylidene]amino]thiophene-3,4-dicarboxylate (3e). Yield: $80 \%$; brown solid, mp $258-260{ }^{\circ} \mathrm{C}$. HPLC purity $97.5 \%\left(t_{\mathrm{R}}=24.98 \mathrm{~min}\right) .{ }^{1} \mathrm{H}$ $\mathrm{NMR}\left(\mathrm{CDCl}_{3}, 400 \mathrm{MHz}, \delta \mathrm{ppm}\right): 8.88$ (d, $\left.J=2.7 \mathrm{~Hz}, 1 \mathrm{H}, \mathrm{Ar}-\mathrm{H}\right)$, $8.29(\mathrm{~s}, 1 \mathrm{H}, \mathrm{N}=\mathrm{CH}), 8.13(\mathrm{dd}, J=8.8 ; 2.7 \mathrm{~Hz}, 1 \mathrm{H}, \mathrm{Ar}-\mathrm{H}), 7.54$ (d, $J=8.8 \mathrm{~Hz}, 1 \mathrm{H}, \mathrm{Ar}-\mathrm{H}), 6.50$ (br.s, $2 \mathrm{H}, \mathrm{NH}_{2}$ ), 4.48 (q, $J=7.1 \mathrm{~Hz}$, $\left.2 \mathrm{H}, \mathrm{OCH}_{2} \mathrm{CH}_{3}\right), 4.27\left(\mathrm{q}, J=7.1 \mathrm{~Hz}, 2 \mathrm{H}, \mathrm{OCH}_{2} \mathrm{CH}_{3}\right), 1.50(\mathrm{t}, J=$ $\left.7.1 \mathrm{~Hz}, 3 \mathrm{H}, \mathrm{OCH}_{2} \boldsymbol{C H}_{3}\right), 1.33\left(\mathrm{t}, J=7.1 \mathrm{~Hz}, 3 \mathrm{H}, \mathrm{OCH}_{2} \boldsymbol{C H}_{3}\right) \cdot{ }^{13} \mathrm{C}$ NMR ( $\left.\mathrm{CDCl}_{3}, 100 \mathrm{MHz}, \delta \mathrm{ppm}\right): 165.12(\mathrm{C}=\mathrm{O}), 164.40(\mathrm{C}=\mathrm{O})$, $161.08\left(\mathrm{NH}_{2} \mathrm{CS}\right), 147.10(\mathrm{Ar}-\mathrm{C}), 145.36(\mathrm{~N}=C \mathrm{H}), 140.61$ (Ar-C), 134.62 (Ar-C), 133.66 (C), 132.75 (C), 131.11 (Ar-CH), 125.09 (Ar$\mathrm{CH}), 123.44$ (Ar-CH), $103.78(\mathrm{C}), 62.11\left(\mathrm{CH}_{2}\right), 60.70\left(\mathrm{CH}_{2}\right), 14.59$ $\left(\mathrm{CH}_{3}\right), 14.28\left(\mathrm{CH}_{3}\right)$. MS (ESI): $m / z 426.5[\mathrm{M}+\mathrm{H}]^{+}$.

Diethyl 2-amino-5-[(E)-[(2-fluoro-5-iodophenyl)methylidene]amino]thiophene-3,4-dicarboxylate (3f). Yield: 81\%; yellow solid, mp 206-208 ${ }^{\circ} \mathrm{C}$. HPLC purity $98.7 \%\left(t_{\mathrm{R}}=26.86 \mathrm{~min}\right) .{ }^{1} \mathrm{H}$ NMR ( $\left.\mathrm{CDCl}_{3}, 400 \mathrm{MHz}, \delta \mathrm{ppm}\right): 8.26$ (dd, $J=6.7 ; 2.3 \mathrm{~Hz}, 1 \mathrm{H}, \mathrm{Ar}-$ $\mathrm{H}), 8.06(\mathrm{~s}, 1 \mathrm{H}, \mathrm{N}=\mathrm{CH}), 7.61$ (ddd, $J=8.6 ; 4.9 ; 2.3 \mathrm{~Hz}, 1 \mathrm{H}, \mathrm{Ar}-\mathrm{H})$, 6.83 (dd, $J=10.2 ; 8.6 \mathrm{~Hz}, 1 \mathrm{H}, \mathrm{Ar}-\mathrm{H}), 6.50$ (br.s, $2 \mathrm{H}, \mathrm{NH}_{2}$ ), 4.45 (q, $\left.J=7.1 \mathrm{~Hz}, 2 \mathrm{H}, \mathrm{OCH}_{2} \mathrm{CH}_{3}\right), 4.25$ (q, $J=7.1 \mathrm{~Hz}, 2 \mathrm{H}, \mathrm{OCH}_{2} \mathrm{CH}_{3}$ ), $1.47\left(\mathrm{t}, J=7.1 \mathrm{~Hz}, 3 \mathrm{H}, \mathrm{OCH}_{2} \boldsymbol{C H}_{3}\right), 1.32(\mathrm{t}, J=7.1 \mathrm{~Hz}, 3 \mathrm{H}$, $\left.\mathrm{OCH}_{2} \boldsymbol{C H}_{3}\right) \cdot{ }^{13} \mathrm{C} \mathrm{NMR}\left(\mathrm{CDCl}_{3}, 100 \mathrm{MHz}, \delta \mathrm{ppm}\right): 165.46(\mathrm{C}=\mathrm{O})$, $164.39(\mathrm{C}=\mathrm{O}), 161.98$ (d, $J=255.0 \mathrm{~Hz}, \mathrm{Ar}-\mathrm{C}), 160.69\left(\mathrm{NH}_{2} \mathrm{CS}\right)$, $143.29(\mathrm{~d}, J=4.6 \mathrm{~Hz}, \mathrm{~N}=C \mathrm{H}), 140.66(\mathrm{~d}, J=8.0 \mathrm{~Hz}, \mathrm{Ar}-\mathrm{CH})$, 136.44 (d, $J=2.4 \mathrm{~Hz}, \operatorname{Ar}-\mathrm{CH}), 133.62$ (C), 131.81 (C), 125.94 (d, $J=10.2 \mathrm{~Hz}, \mathrm{Ar}-\mathrm{C}$ ), 118.01 (d, $J=21.9 \mathrm{~Hz}, \mathrm{Ar}-\mathrm{CH}), 103.23(\mathrm{C})$, $87.83(\mathrm{~d}, J=3.6 \mathrm{~Hz}, \mathrm{Ar}-\mathrm{C}), 61.84\left(\mathrm{CH}_{2}\right), 60.50\left(\mathrm{CH}_{2}\right), 14.56\left(\mathrm{CH}_{3}\right)$, $14.29\left(\mathrm{CH}_{3}\right)$. MS (ESI): $m / z 491[\mathrm{M}+\mathrm{H}]^{+}$.

Diethyl 2-amino-5-[(E)-[(4-chloro-3-fluorophenyl)methylidene]amino]thiophene-3,4-dicarboxylate (3g). Yield: $83.5 \%$; orange solid, $\mathrm{mp} 208-210{ }^{\circ} \mathrm{C}$. HPLC purity $96.2 \%\left(t_{\mathrm{R}}=25.68\right.$ $\min ) .{ }^{1} \mathrm{H} \mathrm{NMR}\left(\mathrm{CDCl}_{3}, 400 \mathrm{MHz}, \delta \mathrm{ppm}\right): 7.84(\mathrm{~s}, 1 \mathrm{H}, \mathrm{N}=\mathrm{CH})$, 7.54 (m, 1H, Ar-H), 7.40 (m, 2H, Ar-H), 6.45 (br.s, 2H, $\mathrm{NH}_{2}$ ), 4.42 (q, $\left.J=7.1 \mathrm{~Hz}, 2 \mathrm{H}, \mathrm{OCH}_{2} \mathrm{CH}_{3}\right), 4.25\left(\mathrm{q}, J=7.1 \mathrm{~Hz}, 2 \mathrm{H}, \mathrm{OCH}_{2} \mathrm{CH}_{3}\right)$, $1.43\left(\mathrm{t}, J=7.1 \mathrm{~Hz}, 3 \mathrm{H}, \mathrm{OCH}_{2} \boldsymbol{C H}_{3}\right), 1.32(\mathrm{t}, J=7.1 \mathrm{~Hz}, 3 \mathrm{H}$, $\left.\mathrm{OCH}_{2} \boldsymbol{C H}_{3}\right) .{ }^{13} \mathrm{C} \mathrm{NMR}\left(\mathrm{CDCl}_{3}, 100 \mathrm{MHz}, \delta \mathrm{ppm}\right): 165.45(\mathrm{C}=\mathrm{O})$,
$164.44(\mathrm{C}=\mathrm{O}), 160.35\left(\mathrm{NH}_{2} \mathrm{CS}\right), 158.40(\mathrm{~d}, J=249.4 \mathrm{~Hz}, \mathrm{Ar}-\mathrm{C})$, 149.81 (d, $J=3.1 \mathrm{~Hz}, \mathrm{~N}=C \mathrm{H}), 136.59$ (d, $J=6.7 \mathrm{~Hz}, \mathrm{Ar}-\mathrm{C}$ ), 133.30 (C), 131.27 (C), 130.95 (Ar-CH), 124.97 (d, $J=3.3 \mathrm{~Hz}$, Ar-CH), 123.55 (d, $J=18.0 \mathrm{~Hz}, \mathrm{Ar}-\mathrm{C}$ ), 115.23 (d, $J=22.4 \mathrm{~Hz}, \mathrm{Ar}-$ $\mathrm{CH}), 103.30(\mathrm{C}), 61.79\left(\mathrm{CH}_{2}\right), 60.52\left(\mathrm{CH}_{2}\right), 14.49\left(\mathrm{CH}_{3}\right), 14.32$ $\left(\mathrm{CH}_{3}\right)$. MS (ESI): $m / z 499.5[\mathrm{M}+\mathrm{H}]^{+}$.

Diethyl 2-amino-5- $[(E)-[(2,3-d i h y d r o b e n z o[b][1,4]$ dioxin-6-yl)methylidene] amino]thiophene-3,4-dicarboxylate (3h). Yield: 79\%; yellow solid, mp 215-217 ${ }^{\circ} \mathrm{C}$. HPLC purity $97.9 \%\left(t_{\mathrm{R}}=\right.$ $24.42 \mathrm{~min}) .{ }^{1} \mathrm{H}$ NMR $\left(\mathrm{CDCl}_{3}, 400 \mathrm{MHz}, \delta \mathrm{ppm}\right): 7.81$ (s, $1 \mathrm{H}, \mathrm{N}=$ $\mathrm{CH}), 7.29(\mathrm{~d}, J=2.0 \mathrm{~Hz}, 1 \mathrm{H}, \mathrm{Ar}-\mathrm{H}), 7.20(\mathrm{dd}, J=8.2 ; 2.0 \mathrm{~Hz}, 1 \mathrm{H}$, Ar-H), 6.85 (d, J=8.2 Hz, 1H, Ar-H), 6.44 (br.s, 2H, $\mathrm{NH}_{2}$ ), 4.41 (q, $\left.J=7.1 \mathrm{~Hz}, 2 \mathrm{H}, \mathrm{OCH}_{2} \mathrm{CH}_{3}\right), 4.25\left(\mathrm{~m}, 6 \mathrm{H}, \mathrm{OCH}_{2} \mathrm{CH}_{3}, \mathrm{OCH}_{2} \boldsymbol{C H}_{2} \mathrm{O}\right)$, $1.42\left(\mathrm{t}, J=7.1 \mathrm{~Hz}, 3 \mathrm{H}, \mathrm{OCH}_{2} \boldsymbol{C H}_{3}\right), 1.30(\mathrm{t}, J=7.1 \mathrm{~Hz}, 3 \mathrm{H}$, $\left.\mathrm{OCH}_{2} \boldsymbol{C H}_{3}\right) \cdot{ }^{13} \mathrm{C} \mathrm{NMR}\left(\mathrm{CDCl}_{3}, 100 \mathrm{MHz}, \delta \mathrm{ppm}\right): 165.86(\mathrm{C}=\mathrm{O})$, $164.53(\mathrm{C}=\mathrm{O}), 159.72\left(\mathrm{NH}_{2} \mathrm{CS}\right), 152.39(\mathrm{~N}=C \mathrm{H}), 146.35(\mathrm{Ar}-\mathrm{C})$, 143.73 (Ar-C), 134.65 (C), 129.70 (Ar-C), 128.69 (C), 122.68 (Ar$\mathrm{CH}), 117.52$ (Ar-CH), $116.86(\mathrm{Ar}-\mathrm{CH}), 102.67(\mathrm{C}), 64.65\left(\mathrm{OCH}_{2}\right)$, $64.20\left(\mathrm{OCH}_{2}\right), 61.60\left(\mathrm{OCH}_{2} \mathrm{CH}_{3}\right), 60.26\left(\mathrm{OCH}_{2} \mathrm{CH}_{3}\right), 14.45\left(\mathrm{CH}_{3}\right)$, $14.29\left(\mathrm{CH}_{3}\right)$. MS (ESI): $m / z 405[\mathrm{M}+\mathrm{H}]^{+}$.

Diethyl 2-amino-5-[(E)-[(3-chloro-2-fluoro-5-(trifluoromethyl)phenyl)methylidene]amino]-thiophene-3,4-dicarboxylate (3i). Yield: $92 \%$; yellow solid, mp $232-234{ }^{\circ} \mathrm{C}$. HPLC purity $97.2 \%$ $\left(t_{\mathrm{R}}=22.96 \mathrm{~min}\right) .{ }^{1} \mathrm{H} \mathrm{NMR}\left(\mathrm{CDCl}_{3}, 400 \mathrm{MHz}, \delta \mathrm{ppm}\right): 8.17(\mathrm{dd}, J=$ $5.8 ; 2.3 \mathrm{~Hz}, 1 \mathrm{H}, \mathrm{Ar}-\mathrm{H}), 8.14(\mathrm{~s}, 1 \mathrm{H}, \mathrm{N}=\mathrm{CH}), 7.67$ (dd, $J=6.5$; $2.3 \mathrm{~Hz}, 1 \mathrm{H}, \mathrm{Ar}-\mathrm{H}$ ), 6.45 (br.s, $2 \mathrm{H}, \mathrm{NH}_{2}$ ), 4.43 (q, $J=7.1 \mathrm{~Hz}, 2 \mathrm{H}$, $\left.\mathrm{OCH}_{2} \mathrm{CH}_{3}\right), 4.27\left(\mathrm{q}, J=7.1 \mathrm{~Hz}, 2 \mathrm{H}, \mathrm{OCH}_{2} \mathrm{CH}_{3}\right), 1.44(\mathrm{t}, J=7.1 \mathrm{~Hz}$, $\left.3 \mathrm{H}, \mathrm{OCH}_{2} \boldsymbol{C H}_{3}\right), 1.32\left(\mathrm{t}, J=7.1 \mathrm{~Hz}, 3 \mathrm{H}, \mathrm{OCH}_{2} \boldsymbol{C H}_{3}\right) \cdot{ }^{13} \mathrm{C} \mathrm{NMR}$ $\left(\mathrm{CDCl}_{3}, 100 \mathrm{MHz}, \delta \mathrm{ppm}\right): 165.14(\mathrm{C}=\mathrm{O}), 164.37(\mathrm{C}=\mathrm{O}), 160.95$ $\left(\mathrm{NH}_{2} \mathrm{CS}\right), 159.00$ (d, $\left.J=259.6 \mathrm{~Hz}, \mathrm{Ar}-\mathrm{CF}\right), 141.93$ (d, $J=4.4 \mathrm{~Hz}$, $\mathrm{N}=C \mathrm{H}$ ), 133.35 (C), 132.84 (C), 128.96 (d, $J=3.8 \mathrm{~Hz}, \mathrm{Ar}-\mathrm{CH})$, 127.79 (d, $J=33.5 \mathrm{~Hz}, \operatorname{Ar}-\mathrm{C}$ ), 126.02 (d, $J=10.8 \mathrm{~Hz}, \mathrm{Ar}-\mathrm{C}$ ), $123.03\left(\mathrm{q}, J=265.0 \mathrm{~Hz}, \mathrm{CF}_{3}\right), 123.44(\mathrm{Ar}-\mathrm{CH}), 122.74(\mathrm{~d}, J=$ $18.2 \mathrm{~Hz}, \mathrm{Ar}-\mathrm{C}), 103.67(\mathrm{C}), 61.92\left(\mathrm{CH}_{2}\right), 60.68\left(\mathrm{CH}_{2}\right), 14.44\left(\mathrm{CH}_{3}\right)$, $14.28\left(\mathrm{CH}_{3}\right)$. MS (ESI): $m / z 467.5[\mathrm{M}+\mathrm{H}]^{+}$.

Diethyl 2-amino-5-[(E)-[(perfluorophenyl)methylidene $]$ amino]thiophene-3,4-dicarboxylate (3j). Yield: $87 \%$; yellow solid, mp 192-194 ${ }^{\circ} \mathrm{C}$. HPLC purity $98.6 \%\left(t_{\mathrm{R}}=25.16 \mathrm{~min}\right) .{ }^{1} \mathrm{H} \mathrm{NMR}\left(\mathrm{CDCl}_{3}\right.$, $400 \mathrm{MHz}, \delta \mathrm{ppm}): 7.95$ (s, 1H, N=CH), 6.54 (br.s, $2 \mathrm{H}, \mathrm{NH}_{2}$ ), 4.39 (q, $\left.J=7.1 \mathrm{~Hz}, 2 \mathrm{H}, \mathrm{OCH}_{2} \mathrm{CH}_{3}\right), 4.25$ (q, $J=7.1 \mathrm{~Hz}, 2 \mathrm{H}, \mathrm{OCH}_{2} \mathrm{CH}_{3}$ ), $1.41\left(\mathrm{t}, J=7.1 \mathrm{~Hz}, 3 \mathrm{H}, \mathrm{OCH}_{2} \boldsymbol{C H}_{3}\right), 1.31(\mathrm{t}, J=7.1 \mathrm{~Hz}, 3 \mathrm{H}$, $\left.\mathrm{OCH}_{2} \boldsymbol{C H}_{3}\right) \cdot{ }^{13} \mathrm{C} \mathrm{NMR}\left(\mathrm{CDCl}_{3}, 100 \mathrm{MHz}, \delta \mathrm{ppm}\right): 165.15(\mathrm{C}=\mathrm{O})$, $164.39(\mathrm{C}=\mathrm{O}), 161.31\left(\mathrm{NH}_{2} \mathrm{CS}\right), 145.87$ (d, $\left.J=257.5 \mathrm{~Hz}, 2 \mathrm{Ar}-\mathrm{C}\right)$, $141.99(\mathrm{~d}, J=258.1 \mathrm{~Hz}, \operatorname{Ar}-\mathrm{C}), 139.05(\mathrm{~d}, J=3.4 \mathrm{~Hz}, \mathrm{~N}=C \mathrm{H})$, 137.68 (d, $J=235.5 \mathrm{~Hz}, 2 \mathrm{Ar}-\mathrm{C}$ ), 133.73 (C), 132.79 (C), 111.50 (ArC), $103.49(\mathrm{C}), 61.97\left(\mathrm{CH}_{2}\right), 60.63\left(\mathrm{CH}_{2}\right), 14.31\left(\mathrm{CH}_{3}\right), 14.26\left(\mathrm{CH}_{3}\right)$. MS (ESI): $m / z 437[\mathrm{M}+\mathrm{H}]^{+}$.

Diethyl 2-amino-5-[(E)-[(5-(4-nitophenyl)furan-2-yl)methylidene]amino]thiophene-3,4-dicarboxylate (3k). Yield: 90\%; dark red solid, mp 197-199 ${ }^{\circ} \mathrm{C}$. HPLC purity $96.7 \%\left(t_{\mathrm{R}}=22.28 \mathrm{~min}\right) .{ }^{1} \mathrm{H}$ NMR ( $\left.\mathrm{CDCl}_{3}, 400 \mathrm{MHz}, \delta \mathrm{ppm}\right): 8.23$ (d, $\left.J=8.9 \mathrm{~Hz}, 2 \mathrm{H}, \mathrm{Ar}-\mathrm{H}\right)$, $7.81(\mathrm{~d}, J=8.9 \mathrm{~Hz}, 2 \mathrm{H}, \operatorname{Ar}-\mathrm{H}), 7.76(\mathrm{~s}, 1 \mathrm{H}, \mathrm{N}=\mathrm{CH}), 6.95(\mathrm{~s}$, $2 \mathrm{H}$, furan-H), 6.54 (br.s, $2 \mathrm{H}, \mathrm{NH}_{2}$ ), 4.44 (q, $J=7.1 \mathrm{~Hz}, 2 \mathrm{H}$, $\left.\mathrm{OCH}_{2} \mathrm{CH}_{3}\right), 4.25\left(\mathrm{q}, J=7.1 \mathrm{~Hz}, 2 \mathrm{H}, \mathrm{OCH}_{2} \mathrm{CH}_{3}\right), 1.47(\mathrm{t}, J=7.1 \mathrm{~Hz}$, $\left.3 \mathrm{H}, \mathrm{OCH}_{2} \boldsymbol{C H}_{3}\right), 1.32\left(\mathrm{t}, J=7.1 \mathrm{~Hz}, 3 \mathrm{H}, \mathrm{OCH}_{2} \boldsymbol{C H}_{3}\right) \cdot{ }^{13} \mathrm{C} \mathrm{NMR}$ $\left(\mathrm{CDCl}_{3}, 100 \mathrm{MHz}, \delta \mathrm{ppm}\right): 165.51(\mathrm{C}=\mathrm{O}), 164.38(\mathrm{C}=\mathrm{O}), 160.23$ $\left(\mathrm{NH}_{2} \mathrm{CS}\right.$ ), 153.77 (furan-C), 153.30 (furan-C), 146.93 (Ar-C), 
$139.70(\mathrm{~N}=C \mathrm{H}), 135.44$ (furan-C), $134.05(\mathrm{C}), 130.69$ (C), 124.61 (2Ar-CH), 124.43 (2Ar-CH), 116.52 (furan-CH), 111.64 (furan$\mathrm{CH}), 103.35$ (C), $61.74\left(\mathrm{CH}_{2}\right), 60.48\left(\mathrm{CH}_{2}\right), 14.47\left(\mathrm{CH}_{3}\right), 14.29$ $\left(\mathrm{CH}_{3}\right)$. MS (ESI): $m / z 458[\mathrm{M}+\mathrm{H}]^{+}$.

Diethyl 2-amino-5-[(E)-[(5-methylfuran-2-yl)methylidene]amino]thiophene-3,4-dicarboxylate (31). Yield: 89\%; yellow solid, mp 152-154 ${ }^{\circ} \mathrm{C}$. HPLC purity $99.0 \%\left(t_{\mathrm{R}}=21.06 \mathrm{~min}\right) .{ }^{1} \mathrm{H}$ $\operatorname{NMR}\left(\mathrm{CDCl}_{3}, 400 \mathrm{MHz}, \delta \mathrm{ppm}\right): 7.71(\mathrm{~s}, 1 \mathrm{H}, \mathrm{N}=\mathrm{CH}), 6.75(\mathrm{~d}, J=$ $3.3 \mathrm{~Hz}, 1 \mathrm{H}$, furan-H), 6.42 (br.s, $2 \mathrm{H}, \mathrm{NH}_{2}$ ), $6.10(\mathrm{~d}, J=3.3 \mathrm{~Hz}$, $1 \mathrm{H}$, furan-H), 4.39 (q, $\left.J=7.1 \mathrm{~Hz}, 2 \mathrm{H}, \mathrm{OCH}_{2} \mathrm{CH}_{3}\right), 4.23$ (q, $J=$ $\left.7.1 \mathrm{~Hz}, 2 \mathrm{H}, \mathrm{OCH}_{2} \mathrm{CH}_{3}\right), 2.36\left(\mathrm{~s}, 3 \mathrm{H}\right.$, furan- $\left.\boldsymbol{C H}_{3}\right), 1.41(\mathrm{t}, J=$ $\left.7.1 \mathrm{~Hz}, 3 \mathrm{H}, \mathrm{OCH}_{2} \boldsymbol{C H}_{3}\right), 1.30\left(\mathrm{t}, J=7.1 \mathrm{~Hz}, 3 \mathrm{H}, \mathrm{OCH}_{2} \boldsymbol{C H}_{3}\right) \cdot{ }^{13} \mathrm{C}$ NMR ( $\left.\mathrm{CDCl}_{3}, 100 \mathrm{MHz}, \delta \mathrm{ppm}\right): 165.69(\mathrm{C}=\mathrm{O}), 164.53(\mathrm{C}=\mathrm{O})$, $159.45\left(\mathrm{NH}_{2} \mathrm{CS}\right.$ ), 156.52 (furan-C), 150.56 (furan-C), $141.37(\mathrm{~N}=$ CH), 135.09 (C), 128.41 (C), 117.00 (furan-CH), 109.20 (furan$\mathrm{CH}$ ), $103.11(\mathrm{C}), 61.61\left(\mathrm{CH}_{2}\right), 60.28\left(\mathrm{CH}_{2}\right), 14.31$ (furan- $\mathrm{CH}_{3}$, $\left.\mathrm{CH}_{3}\right), 14.09\left(\mathrm{CH}_{3}\right)$. MS (ESI): $m / z 351[\mathrm{M}+\mathrm{H}]^{+}$.

Diethyl 2-amino-5-[(E)-[(4,5-dimethylfuran-2-yl)methylidene]amino]thiophene-3,4-dicarboxylate (3m). Yield: 86\%; brown solid, mp $182-184{ }^{\circ} \mathrm{C}$. HPLC purity $97.4 \%\left(t_{\mathrm{R}}=22.59 \mathrm{~min}\right) .{ }^{1} \mathrm{H}$ NMR $\left(\mathrm{CDCl}_{3}, 400 \mathrm{MHz}, \delta \mathrm{ppm}\right): 7.68(\mathrm{~s}, 1 \mathrm{H}, \mathrm{N}=\mathrm{CH}), 6.67(\mathrm{~s}, 1 \mathrm{H}$, furan-H), 6.34 (br.s, $2 \mathrm{H}, \mathrm{NH}_{2}$ ), 4.39 (q, $J=7.1 \mathrm{~Hz}, 2 \mathrm{H}, \mathrm{OCH}_{2} \mathrm{CH}_{3}$ ), 4.23 (q, $\left.J=7.1 \mathrm{~Hz}, 2 \mathrm{H}, \mathrm{OCH}_{2} \mathrm{CH}_{3}\right), 2.27$ (s, 3H, furan- $\left.\mathbf{C H}_{3}\right), 1.96$ $\left(\mathrm{s}, 3 \mathrm{H}\right.$, furan- $\left.\boldsymbol{C H}_{3}\right), 1.40\left(\mathrm{t}, J=7.1 \mathrm{~Hz}, 3 \mathrm{H}, \mathrm{OCH}_{2} \boldsymbol{C H}_{3}\right), 1.30(\mathrm{t}, J=$ $\left.7.1 \mathrm{~Hz}, 3 \mathrm{H}, \mathrm{OCH}_{2} \boldsymbol{C H}_{3}\right) .{ }^{13} \mathrm{C} \mathrm{NMR}\left(\mathrm{CDCl}_{3}, 100 \mathrm{MHz}, \delta \mathrm{ppm}\right)$ : $165.67(\mathrm{C}=\mathrm{O}), 164.59(\mathrm{C}=\mathrm{O}), 159.23\left(\mathrm{NH}_{2} \mathrm{CS}\right), 152.54$ (furan-C), 149.29 (furan-C), $141.56(\mathrm{~N}=\mathrm{CH}), 135.41$ (C), 128.02 (C), 119.08 (furan-CH), 117.96 (furan-C), 103.24 (C), $61.59\left(\mathrm{CH}_{2}\right), 60.30$ $\left(\mathrm{CH}_{2}\right), 14.35\left(2 \mathrm{CH}_{3}\right), 12.00$ (furan- $\left.\mathrm{CH}_{3}\right), 9.91$ (furan- $\left.\mathrm{CH}_{3}\right) . \mathrm{MS}$ (ESI): $m / z 365[\mathrm{M}+\mathrm{H}]^{+}$.

Diethyl 2-amino-5-[(E)-[(5-nitrothiophen-2-yl)methylidene]amino]thiophene-3,4-dicarboxylate (3n). Yield: 94\%; dark red solid, mp 187-189 ${ }^{\circ} \mathrm{C}$. HPLC purity $99.8 \%\left(t_{\mathrm{R}}=22.70 \mathrm{~min}\right) .{ }^{1} \mathrm{H}$ NMR $\left(\mathrm{CDCl}_{3}, 400 \mathrm{MHz}, \delta \mathrm{ppm}\right): 7.92(\mathrm{~s}, 1 \mathrm{H}, \mathrm{N}=\mathrm{CH}), 7.84(\mathrm{~d}, J=$ $4.3 \mathrm{~Hz}, 1 \mathrm{H}$, thiophene-H), 7.19 (d, $J=4.3 \mathrm{~Hz}, 1 \mathrm{H}$, thiophene-H), 6.54 (br.s, $2 \mathrm{H}, \mathrm{NH}_{2}$ ), 4.43 (q, $J=7.1 \mathrm{~Hz}, 2 \mathrm{H}, \mathrm{OCH}_{2} \mathrm{CH}_{3}$ ), 4.26 (q, $\left.J=7.1 \mathrm{~Hz}, 2 \mathrm{H}, \mathrm{OCH}_{2} \mathrm{CH}_{3}\right), 1.46\left(\mathrm{t}, J=7.1 \mathrm{~Hz}, 3 \mathrm{H}, \mathrm{OCH}_{2} \boldsymbol{C H}_{3}\right)$, $1.32\left(\mathrm{t}, J=7.1 \mathrm{~Hz}, 3 \mathrm{H}, \mathrm{OCH}_{2} \boldsymbol{C H}_{3}\right) \cdot{ }^{13} \mathrm{C} \mathrm{NMR}\left(\mathrm{CDCl}_{3}, 100 \mathrm{MHz}\right.$, $\delta$ ppm): $164.87(\mathrm{C}=\mathrm{O}), 164.29(\mathrm{C}=\mathrm{O}), 161.09\left(\mathrm{NH}_{2} \mathrm{CS}\right), 153.19$ (thiophene-C), 148.93 (thiophene-C), $142.84(\mathrm{~N}=\mathrm{CH}), 133.49$ (C), 132.07 (C), 129.01 (thiophene-CH), 128.20 (thiophene- $\mathrm{CH}$ ), $103.88(\mathrm{C}), 62.04\left(\mathrm{CH}_{2}\right), 60.74\left(\mathrm{CH}_{2}\right), 14.62\left(\mathrm{CH}_{3}\right), 14.28\left(\mathrm{CH}_{3}\right)$. MS (ESI): $m / z 398[\mathrm{M}+\mathrm{H}]^{+}$.

Diethyl 2-amino-5-[(E)-[(4,5-dimethylthiophen-2-yl)methylidene]amino]thiophene-3,4-dicarboxylate (3o). Yield: $88 \%$; brown solid, mp 210-212 ${ }^{\circ} \mathrm{C}$. HPLC purity $98.9 \%\left(t_{\mathrm{R}}=24.64\right.$ $\min ) .{ }^{1} \mathrm{H} \mathrm{NMR}\left(\mathrm{CDCl}_{3}, 400 \mathrm{MHz}, \delta \mathrm{ppm}\right): 7.94(\mathrm{~s}, 1 \mathrm{H}, \mathrm{N}=\mathrm{CH})$, 7.05 (s, 1H, thiophene-H), 6.24 (br.s, $2 \mathrm{H}, \mathrm{NH}_{2}$ ), 4.40 (q, $J=$ $\left.7.1 \mathrm{~Hz}, 2 \mathrm{H}, \mathrm{OCH}_{2} \mathrm{CH}_{3}\right), 4.23\left(\mathrm{q}, J=7.1 \mathrm{~Hz}, 2 \mathrm{H}, \mathrm{OCH}_{2} \mathrm{CH}_{3}\right), 2.36$ $\left(\mathrm{s}, 3 \mathrm{H}\right.$, thiophene- $\left.\boldsymbol{C H}_{3}\right), 2.11\left(\mathrm{~s}, 3 \mathrm{H}\right.$, thiophene- $\left.\boldsymbol{H}_{3}\right), 1.43(\mathrm{t}, J=$ $\left.7.1 \mathrm{~Hz}, 3 \mathrm{H}, \mathrm{OCH}_{2} \boldsymbol{C H}_{3}\right), 1.30\left(\mathrm{t}, J=7.1 \mathrm{~Hz}, 3 \mathrm{H}, \mathrm{OCH}_{2} \boldsymbol{C H}_{3}\right) \cdot{ }^{13} \mathrm{C}$ NMR ( $\left.\mathrm{CDCl}_{3}, 100 \mathrm{MHz}, \delta \mathrm{ppm}\right): 165.57(\mathrm{C}=\mathrm{O}), 164.59(\mathrm{C}=\mathrm{O})$, $159.17\left(\mathrm{NH}_{2} \mathrm{CS}\right), 146.50(\mathrm{~N}=\boldsymbol{C H}), 139.98$ (thiophene-C), 137.58 (thiophene-C), 134.72 (C), 134.63 (thiophene-CH), 134.51 (thiophene-C), 128.30 (C), 103.18 (C), $61.57\left(\mathrm{CH}_{2}\right), 60.34\left(\mathrm{CH}_{2}\right)$, $14.59\left(\mathrm{CH}_{3}\right), 14.30\left(\mathrm{CH}_{3}\right), 14.03$ (thiophene- $\left.\mathrm{CH}_{3}\right), 13.69$ (thiophene- $\mathrm{CH}_{3}$ ). MS (ESI): $m / z 381[\mathrm{M}+\mathrm{H}]^{+}$.
Diethyl 2-amino-5-[(E)-[(1-methyl-1H-pyrazol-4-yl)methylidene]amino]thiophene-3,4-dicarboxylate (3p). Yield: $91 \%$; yellow solid, mp $175-177{ }^{\circ} \mathrm{C}$. HPLC purity $99.7 \%\left(t_{\mathrm{R}}=16.29\right.$ min). ${ }^{1} \mathrm{H} \mathrm{NMR}\left(\mathrm{CDCl}_{3}, 400 \mathrm{MHz}, \delta \mathrm{ppm}\right): 7.88(\mathrm{~s}, 1 \mathrm{H}, \mathrm{N}=\mathrm{CH})$, 7.79 (s, 1H, pyrazole-H), 7.71 (s, 1H, pyrazole- $\mathrm{H}), 6.40$ (br.s, $2 \mathrm{H}$, $\left.\mathrm{NH}_{2}\right), 4.38\left(\mathrm{q}, J=7.1 \mathrm{~Hz}, 2 \mathrm{H}, \mathrm{OCH}_{2} \mathrm{CH}_{3}\right), 4.24(\mathrm{q}, J=7.1 \mathrm{~Hz}, 2 \mathrm{H}$, $\left.\mathrm{OCH}_{2} \mathrm{CH}_{3}\right), 3.90\left(\mathrm{~s}, 3 \mathrm{H}, \mathrm{N}-\boldsymbol{C H}_{3}\right), 1.40(\mathrm{t}, J=7.1 \mathrm{~Hz}, 3 \mathrm{H}$, $\left.\mathrm{OCH}_{2} \boldsymbol{C H}_{3}\right), 1.30\left(\mathrm{t}, J=7.1 \mathrm{~Hz}, 3 \mathrm{H}, \mathrm{OCH}_{2} \boldsymbol{C H}_{3}\right) \cdot{ }^{13} \mathrm{C} \mathrm{NMR}\left(\mathrm{CDCl}_{3}\right.$, $100 \mathrm{MHz}, \delta \mathrm{ppm}): 165.81(\mathrm{C}=\mathrm{O}), 164.55(\mathrm{C}=\mathrm{O}), 159.26$ $\left(\mathrm{NH}_{2} \mathrm{CS}\right), 145.73(\mathrm{~N}=\boldsymbol{C H}), 139.82$ (pyrazole-CH), $135.08(\mathrm{C})$, 130.89 (pyrazole-CH), 127.69 (C), 121.24 (pyrazole-C), 102.75 (C), $61.52\left(\mathrm{CH}_{2}\right), 60.26\left(\mathrm{CH}_{2}\right), 39.33\left(\mathrm{~N}-\mathrm{CH}_{3}\right), 14.42\left(\mathrm{CH}_{3}\right), 14.32$ $\left(\mathrm{CH}_{3}\right)$. MS (ESI): $m / z 351[\mathrm{M}+\mathrm{H}]^{+}$.

General procedure for the synthesis of compounds $4 a-d$ and 5a-c

Method (a). DDTD (1 mmol) and aromatic acid anhydride $(1.2 \mathrm{mmol}$ ) were dissolved in $10 \mathrm{~mL}$ dry benzene or chloroform. The mixture was refluxed for 2-3 hours after the addition of TEA ( $2 \mathrm{mmol}$ ). $5 \mathrm{~mL}$ water was added to the cooled mixture and extracted with chloroform $(3 \times 10 \mathrm{~mL})$. The combined organic layers were washed with brine, dried over $\mathrm{Na}_{2} \mathrm{SO}_{4}$ and evaporated under reduced pressure. The obtained solid was filtered, washed with petroleum ester, dried and recrystallized from ethanol.

Method (b). DDTD (1 mmol) and aromatic acid anhydride $(1.2 \mathrm{mmol})$ were dissolved in $10 \mathrm{~mL}$ dry pyridine at $0{ }^{\circ} \mathrm{C}$ and stirred for 4 hours at room temperature. Water $(5 \mathrm{~mL})$ and $5 \%$ $\mathrm{NH}_{4} \mathrm{OH}$ were added to the reaction mixture. The solid formed was filtered, dried and recrystallized from ethanol.

Diethyl 2,5-bis(3-methylbenzamido)thiophene-3,4-dicarboxylate (4a). Yield: $93 \%$; green solid, mp $158-160{ }^{\circ} \mathrm{C}$. HPLC purity $96.9 \%\left(t_{\mathrm{R}}=28.16 \mathrm{~min}\right) .{ }^{1} \mathrm{H} \mathrm{NMR}\left(\mathrm{CDCl}_{3}, 600 \mathrm{MHz}, \delta \mathrm{ppm}\right)$ : 11.36 (s, 2H, NH), 7.83 (s, 2H, Ar-H), 7.76 (d, J=6.4 Hz, 2H, Ar$\mathrm{H}), 7.41(\mathrm{~m}, 4 \mathrm{H}, \mathrm{Ar}-\mathrm{H}), 4.40\left(\mathrm{q}, J=7.2 \mathrm{~Hz}, 4 \mathrm{H}, \mathrm{OCH}_{2} \mathrm{CH}_{3}\right), 2.46$ $\left(\mathrm{s}, 6 \mathrm{H}, \mathrm{Ar}_{-} \mathrm{CH}_{3}\right), 1.39\left(\mathrm{t}, J=7.2 \mathrm{~Hz}, 6 \mathrm{H}, \mathrm{OCH}_{2} \boldsymbol{C H}_{3}\right) \cdot{ }^{13} \mathrm{C} \mathrm{NMR}$ $\left(\mathrm{CDCl}_{3}, 100 \mathrm{MHz}, \delta \mathrm{ppm}\right): 166.17(2 \mathrm{C}=\mathrm{O}), 164.06(2 \mathrm{HNC}=\mathrm{O})$, 142.63 (2HNCS), 139.08 (2Ar-C), 133.60 (2Ar-CH), 132.20 (2ArC), 128.96 (2Ar-CH), 128.52 (2Ar-CH), 124.50 (2Ar-CH), 108.58 (2C), $61.39\left(2 \mathrm{CH}_{2}\right), 21.60\left(2 \mathrm{Ar}-\mathrm{CH}_{3}\right), 14.43\left(2 \mathrm{CH}_{3}\right) . \mathrm{MS}$ (ESI): $\mathrm{m} /$ $z 495[\mathrm{M}+\mathrm{H}]^{+}$.

Diethyl 2,5-bis(3,5-bis(trifluoromethyl)benzamido)thiophene3,4-dicarboxylate (4b). Yield: 95\%; white solid, mp 203$205{ }^{\circ} \mathrm{C}$. HPLC purity $97.6 \%\left(t_{\mathrm{R}}=32.53 \mathrm{~min}\right) .{ }^{1} \mathrm{H} \mathrm{NMR}\left(\mathrm{CDCl}_{3}\right.$, $600 \mathrm{MHz}, \delta \mathrm{ppm}): 11.72$ (s, 2H, NH), 8.43 (s, 4H, Ar-H), 8.12 (s, $2 \mathrm{H}, \mathrm{Ar}-\mathrm{H}), 4.44$ (q, $\left.J=7.2 \mathrm{~Hz}, 4 \mathrm{H}, \mathrm{OCH}_{2} \mathrm{CH}_{3}\right), 1.41$ (t, $J=7.2 \mathrm{~Hz}$, $\left.6 \mathrm{H}, \mathrm{OCH}_{2} \boldsymbol{C H}_{3}\right) .{ }^{13} \mathrm{C} \mathrm{NMR}\left(\mathrm{CDCl}_{3}, 100 \mathrm{MHz}, \delta \mathrm{ppm}\right): 165.97$ $(2 \mathrm{C}=\mathrm{O}), 161.11$ (2HNC=O), 141.83 (2HNCS), 134.39 (2Ar-C), 132.96 (q, $J=34.0 \mathrm{~Hz}, 4 \mathrm{Ar}-\mathrm{C}), 127.86$ (4Ar-CH), 126.33 (2Ar$\mathrm{CH}), 122.90$ (d, $\left.J=272.9 \mathrm{~Hz}, 4 \mathrm{CF}_{3}\right), 110.08(2 \mathrm{C}), 61.96\left(2 \mathrm{CH}_{2}\right)$, $14.36\left(2 \mathrm{CH}_{3}\right)$. MS (ESI): $m / z 739[\mathrm{M}+\mathrm{H}]^{+}$.

Diethyl 2,5-bis(3,4,5-trifluorobenzamido)thiophene-3,4dicarboxylate (4c). Yield: 95\%; green solid, mp $164-166{ }^{\circ} \mathrm{C}$. HPLC purity $99.1 \%\left(t_{\mathrm{R}}=30.82 \mathrm{~min}\right) .{ }^{1} \mathrm{H} \mathrm{NMR}\left(\mathrm{CDCl}_{3}, 600 \mathrm{MHz}\right.$, $\delta$ ppm): 11.52 (s, 2H, NH), 7.65 (d, J=6.4 Hz, 2H, Ar-H), 7.64 (d, $J=6.4 \mathrm{~Hz}, 2 \mathrm{H}, \mathrm{Ar}-\mathrm{H}), 4.42\left(\mathrm{q}, J=7.2 \mathrm{~Hz}, 4 \mathrm{H}, \mathrm{OCH}_{2} \mathrm{CH}_{3}\right), 1.40(\mathrm{t}$, 
$\left.J=7.2 \mathrm{~Hz}, 6 \mathrm{H}, \mathrm{OCH}_{2} \boldsymbol{C H}_{3}\right) .{ }^{13} \mathrm{C} \mathrm{NMR}\left(\mathrm{CDCl}_{3}, 100 \mathrm{MHz}, \delta \mathrm{ppm}\right)$ : $166.20(2 \mathrm{C}=\mathrm{O}), 160.80(2 \mathrm{HNC}=\mathrm{O}), 151.57$ (dd, $J=253.3$; $10.3 \mathrm{~Hz}, 4 \mathrm{Ar}-\mathrm{CF}$ ), 142.99 (dt, $J=259.0 ; 11.4 \mathrm{~Hz}, 2 \mathrm{Ar}-\mathrm{CF}$ ), 142.11 (2HNCS), 128.14 (Ar-C), 112.45 (dd, $J=16.3 ; 6.6 \mathrm{~Hz}, 4 \mathrm{Ar}-\mathrm{CH}$ ), 109.43 (2C), $61.84\left(2 \mathrm{CH}_{2}\right), 14.39\left(2 \mathrm{CH}_{3}\right) . \mathrm{MS}$ (ESI): $m / z 575$ $[\mathrm{M}+\mathrm{H}]^{+}$.

Diethyl 2,5-bis(3,4,5-trimethoxybenzamido)thiophene-3,4dicarboxylate (4d). Yield: 92\%; white solid, mp 216-218 ${ }^{\circ} \mathrm{C}$. HPLC purity $98.0 \%\left(t_{\mathrm{R}}=27.92 \mathrm{~min}\right) .{ }^{1} \mathrm{H} \mathrm{NMR}\left(\mathrm{CDCl}_{3}, 600 \mathrm{MHz}\right.$, $\delta$ ppm): 11.42 (s, 2H, NH), $7.23(\mathrm{~s}, 4 \mathrm{H}, \mathrm{Ar}-\mathrm{H}), 4.38(\mathrm{q}, J=7.2 \mathrm{~Hz}$, $\left.4 \mathrm{H}, \mathrm{OCH}_{2} \mathrm{CH}_{3}\right), 3.97\left(\mathrm{~s}, 12 \mathrm{H}, \mathrm{OCH}_{3}\right), 3.94\left(\mathrm{~s}, 6 \mathrm{H}, \mathrm{OCH}_{3}\right), 1.38(\mathrm{t}$, $\left.J=7.2 \mathrm{~Hz}, 6 \mathrm{H}, \mathrm{OCH}_{2} \boldsymbol{C H}_{3}\right) \cdot{ }^{13} \mathrm{C} \mathrm{NMR}\left(\mathrm{CDCl}_{3}, 100 \mathrm{MHz}, \delta \mathrm{ppm}\right)$ : $166.29(2 \mathrm{C}=\mathrm{O}), 163.26(2 \mathrm{HNC}=\mathrm{O}), 153.58$ (4Ar-C), 142.64 (2HNCS), 142.17 (2Ar-C), 127.46 (2Ar-C), 108.58 (2C), 105.05 $(4 \mathrm{Ar}-\mathrm{CH}), 61.39\left(2 \mathrm{CH}_{2}\right), 61.15\left(2 \mathrm{OCH}_{3}\right), 56.53\left(4 \mathrm{OCH}_{3}\right), 14.41$ $\left(2 \mathrm{CH}_{3}\right)$. MS (ESI): $m / z 647[\mathrm{M}+\mathrm{H}]^{+}$.

Diethyl 2-amino-5-(3-nitrobenzamido)thiophene-3,4dicarboxylate (5a). Yield: 96\%; yellow solid, mp 198-200 ${ }^{\circ} \mathrm{C}$. HPLC purity $98.5 \%\left(t_{\mathrm{R}}=22.35 \mathrm{~min}\right) .{ }^{1} \mathrm{H} \mathrm{NMR}\left(\mathrm{CDCl}_{3}, 600 \mathrm{MHz}\right.$, $\delta \mathrm{ppm}): 11.65(\mathrm{~s}, 1 \mathrm{H}, \mathrm{NH}), 8.83(\mathrm{t}, J=2.0 \mathrm{~Hz}, 1 \mathrm{H}, \mathrm{Ar}-\mathrm{H}), 8.44$ (ddd, $J=8.0 ; 2.0 ; 0.9 \mathrm{~Hz}, 1 \mathrm{H}, \mathrm{Ar}-\mathrm{H}), 8.24(\mathrm{dt}, J=8.0 ; 1.1 \mathrm{~Hz}, 1 \mathrm{H}$, $\operatorname{Ar}-\mathrm{H}$ ), 7.74 (t, $J=8.0 \mathrm{~Hz}, 1 \mathrm{H}, \mathrm{Ar}-\mathrm{H}), 5.56$ (br.s, $2 \mathrm{H}, \mathrm{NH}_{2}$ ), 4.37 (q, $\left.J=7.1 \mathrm{~Hz}, 2 \mathrm{H}, \mathrm{OCH}_{2} \mathrm{CH}_{3}\right), 4.30\left(\mathrm{q}, J=7.1 \mathrm{~Hz}, 2 \mathrm{H}, \mathrm{OCH}_{2} \mathrm{CH}_{3}\right)$, $1.37\left(\mathrm{t}, J=7.1 \mathrm{~Hz}, 3 \mathrm{H}, \mathrm{OCH}_{2} \boldsymbol{C H}_{3}\right), 1.34(\mathrm{t}, J=7.1 \mathrm{~Hz}, 3 \mathrm{H}$, $\left.\mathrm{OCH}_{2} \boldsymbol{C H}_{3}\right) \cdot{ }^{13} \mathrm{C} \mathrm{NMR}\left(\mathrm{CDCl}_{3}, 100 \mathrm{MHz}, \delta \mathrm{ppm}\right): 166.20(\mathrm{C}=\mathrm{O})$, $165.24(\mathrm{C}=\mathrm{O}), 161.36(\mathrm{HNC}=\mathrm{O}), 154.73\left(\mathrm{NH}_{2} \mathrm{CS}\right), 148.82$ (HNCS), 134.63 (Ar-C), 134.05 (Ar-C), 132.73 (Ar-CH), 130.37 (Ar$\mathrm{CH}), 127.14$ (Ar-CH), 122.97 (Ar-CH), 111.22 (C), 102.37 (C), $61.51\left(\mathrm{CH}_{2}\right), 60.48\left(\mathrm{CH}_{2}\right), 14.57\left(\mathrm{CH}_{3}\right), 14.34\left(\mathrm{CH}_{3}\right) . \mathrm{MS}$ (ESI): $\mathrm{m} / z$ $408[\mathrm{M}+\mathrm{H}]^{+}$.

Diethyl 2-amino-5-(3,4,5-trimethoxybenzamido)thiophene3,4-dicarboxylate (5b). Yield: 53\%; green solid, mp 138$140{ }^{\circ} \mathrm{C}$. HPLC purity $99.6 \%\left(t_{\mathrm{R}}=21.95 \mathrm{~min}\right) .{ }^{1} \mathrm{H} \mathrm{NMR}\left(\mathrm{CDCl}_{3}\right.$, $400 \mathrm{MHz}, \delta \mathrm{ppm}): 11.41$ (s, 1H, NH), 7.18 (s, 2H, Ar-H), 5.54 (br.s, $2 \mathrm{H}, \mathrm{NH}_{2}$ ), 3.95 (s, 6H, $\mathrm{OCH}_{3}$ ), $3.92\left(\mathrm{~s}, 3 \mathrm{H}, \mathrm{OCH}_{3}\right), 4.34$ (q, $\left.J=7.1 \mathrm{~Hz}, 2 \mathrm{H}, \mathrm{OCH}_{2} \mathrm{CH}_{3}\right), 4.28\left(\mathrm{q}, J=7.1 \mathrm{~Hz}, 2 \mathrm{H}, \mathrm{OCH}_{2} \mathrm{CH}_{3}\right)$, $1.35\left(\mathrm{t}, J=7.1 \mathrm{~Hz}, 3 \mathrm{H}, \mathrm{OCH}_{2} \boldsymbol{C H}_{3}\right), 1.33(\mathrm{t}, J=7.1 \mathrm{~Hz}, 3 \mathrm{H}$, $\left.\mathrm{OCH}_{2} \boldsymbol{C H}_{3}\right) \cdot{ }^{13} \mathrm{C} \mathrm{NMR}\left(\mathrm{CDCl}_{3}, 100 \mathrm{MHz}, \delta \mathrm{ppm}\right): 166.20(\mathrm{C}=\mathrm{O})$, $165.38(\mathrm{C}=\mathrm{O}), 163.48(\mathrm{HNC}=\mathrm{O}), 154.50\left(\mathrm{NH}_{2} \mathrm{CS}\right), 153.56(2 \mathrm{Ar}-$ C), 142.14 (HNCS), 135.61 (Ar-C), 127.34 (Ar-C), 110.14 (C), 104.88 (2Ar-CH), 102.20 (C), $61.18\left(\mathrm{CH}_{2}\right), 61.13\left(\mathrm{OCH}_{3}\right), 60.34$ $\left(\mathrm{CH}_{2}\right), 56.49\left(2 \mathrm{OCH}_{3}\right), 14.55\left(\mathrm{CH}_{3}\right), 14.35\left(\mathrm{CH}_{3}\right) . \mathrm{MS}(\mathrm{ESI}): \mathrm{m} / z$ $453[\mathrm{M}+\mathrm{H}]^{+}$.

Diethyl 2-amino-5-(6-chloronicotinamido)thiophene-3,4dicarboxylate (5c). Yield: 97.5\%; yellow solid, mp 204-206 ${ }^{\circ} \mathrm{C}$. HPLC purity $98.9 \%\left(t_{\mathrm{R}}=21.24 \mathrm{~min}\right) .{ }^{1} \mathrm{H} \mathrm{NMR}\left(\mathrm{CDCl}_{3}, 600 \mathrm{MHz}\right.$, $\delta \mathrm{ppm}): 11.57$ (s, 1H, NH), 8.98 (d, $J=2.7 \mathrm{~Hz}, 1 \mathrm{H}, \mathrm{Ar}-\mathrm{H}), 8.17$ (dd, $J=8.4 ; 2.7 \mathrm{~Hz}, 1 \mathrm{H}, \mathrm{Ar}-\mathrm{H}), 7.49$ (d, $J=8.4 \mathrm{~Hz}, 1 \mathrm{H}, \mathrm{Ar}-\mathrm{H}), 5.52$ (br.s, 2H, $\mathrm{NH}_{2}$ ), 4.35 (q, $J=7.1 \mathrm{~Hz}, 2 \mathrm{H}, \mathrm{OCH}_{2} \mathrm{CH}_{3}$ ), 4.29 (q, $J=$ $\left.7.1 \mathrm{~Hz}, 2 \mathrm{H}, \mathrm{OCH}_{2} \mathrm{CH}_{3}\right), 1.36\left(\mathrm{t}, J=7.1 \mathrm{~Hz}, 3 \mathrm{H}, \mathrm{OCH}_{2} \boldsymbol{C H}_{3}\right), 1.34(\mathrm{t}$, $\left.J=7.1 \mathrm{~Hz}, 3 \mathrm{H}, \mathrm{OCH}_{2} \boldsymbol{C H}_{3}\right) \cdot{ }^{13} \mathrm{C} \mathrm{NMR}\left(\mathrm{CDCl}_{3}, 100 \mathrm{MHz}, \delta \mathrm{ppm}\right)$ : $166.25(\mathrm{C}=\mathrm{O}), \quad 165.24(\mathrm{C}=\mathrm{O}), 162.15 \quad(\mathrm{HNC}=\mathrm{O}), 160.95$ $\left(\mathrm{NH}_{2} \mathrm{CS}\right.$ ), 155.58 (HNCS), 154.70 (Ar-C), 149.11 (Ar-CH), 137.63 (Ar-CH), 134.52 (Ar-C), 126.95 (C), 124.73 (Ar-CH), 102.30 (C), $61.49\left(\mathrm{CH}_{2}\right), 60.47\left(\mathrm{CH}_{2}\right), 14.56\left(\mathrm{CH}_{3}\right), 14.33\left(\mathrm{CH}_{3}\right)$. MS (ESI): $\mathrm{m} / z$ $398.5[\mathrm{M}+\mathrm{H}]^{+}$.

\section{Biology}

Materials. Doxorubicin and trypsin were purchased from BBI Inc. (Shanghai, China), ampicillin from Sigma Chemicals Co., amphotericin B. from AMRESCO LLC, ribavirin from Xinxiang Pharmaceutical Co., Ltd. (batch 20081227) and oseltamivir from Chinese Academy of Food and Drug (batch 101096-200901). The human cancer cell prostate (PC-3), lung (A549), colon (HCT-15), breast (T47D) and human embryonic kidney (HEK-293) lines were obtained from Chinese Type Culture Collection, CAS (Shanghai, China). Madin-Darby canine kidney cells (MDCK) were purchased from the American Type Culture Collection (ATCC).

Cell cultures. Human PC-3 cells and HCT-15 cells were grown in DMEM/F12 medium (Gibco), human T47D cells and A549 cells were grown in Dulbecco's modified Eagle's medium (DMEM) with $4.5 \mathrm{~g} \mathrm{~L}^{-1}$ glucose and $0.37 \%$ sodium bicarbonate (Gibco, Rockville, MD, USA). All cell culture media contained with $10 \%$ FBS and antibiotic mix $(1 \times 100 \mu \mathrm{M}$ penicillin $\mathrm{A}$ and $100 \mu \mathrm{M}$ of streptomycin) and were grown at $37{ }^{\circ} \mathrm{C}$ in a humidified incubator (Binder, Germany) containing 95\% air/5\% $\mathrm{CO}_{2}$ and have been fed every 3-4 days. ${ }^{27}$

Proliferation assays. All compounds were dissolved in DMSO in a stock concentration of 10 mM. PC-3, A549, HCT-15 or T47D cells grown in the logarithmic phase were separately seeded in aliquots of $200 \mu \mathrm{L}$ in 96-well plates at a density of $5 \times 10^{3}$ cells per well. The cells were grown for $24 \mathrm{~h}$ in a humidified incubator (Binder, Germany) at $37{ }^{\circ} \mathrm{C}$ with $95 \%$ humidity and $5 \% \mathrm{CO}_{2}$. Thereafter, the cells were treated with $1,10,25$ and $30 \mu \mathrm{M}$ of compounds (1-5) for $48 \mathrm{~h}$. Then $20 \mu \mathrm{L}$ MTT $\left(5 \mathrm{mg} \mathrm{mL}^{-1}\right)$ was added to each well and the plates incubated at $37^{\circ} \mathrm{C}$. Four hours later, the supernatant was removed and $200 \mu \mathrm{L}$ of DMSO were added to each well and the multiwell plates shaken for $10 \mathrm{~min}$ to dissolve the crystals. Absorbance was read at a wavelength of $540 \mathrm{~nm}$ using an enzyme-linked immunosorbent assay reader (SpectraMax M5, Molecular Devices, U.S.). The IC $_{50}$ values were calculated with the inhibition rate. Inhibition rate $=(\mathrm{OD}$ value of control group-experiment group)/(OD value of control group - OD value of blank group). ${ }^{27}$

Cell cycle assay. For cell cycle analysis, approximately $5 \times 10^{5}$ log phase A549 cells were seeded in six-well plates in triplicate and treated with various concentrations of selected compounds (DOX, 3j, 3n, 3p) for $12 \mathrm{~h}$. Then, samples were suspended in absolute ice-cold ethanol and kept overnight. The next day, samples were stained with $30 \mu \mathrm{L}$ PI and $0.3 \mu \mathrm{L}$ RNase A at room temperature in the absence of light for at least $0.5 \mathrm{~h}$. The stained cells were filtered through a $30 \mathrm{~mm}$ nylon mesh (Spectrum, Los Angeles, CA, U.S.).

Cellular apoptosis analysis by flow cytometry. A549 cells were seeded in six-well plates at a density of $5 \times 10^{5}$ cells per well and allowed to attach for $24 \mathrm{~h}$. Further, cells were incubated with $\operatorname{DOX}(1,2.5$ and $5 \mu \mathrm{M})$ or with selected compounds $3 \mathbf{j}$ (1, 2.5, $5 \mu \mathrm{M}), 3 \mathrm{n}(0.75,1.5,2 \mu \mathrm{M})$ and $3 \mathbf{p}(5,10,20 \mu \mathrm{M})$ for $12 \mathrm{~h}$. Treated cells were collected, washed twice with ice-cold PBS, centrifuged at $200 \times g$ for $5 \mathrm{~min}$. and re-suspended in $100 \mu \mathrm{L}$ of Annexin-V-FLOUS (Roche, Germany) for $15 \mathrm{~min}$. Incubation at RT in the dark followed according to the manufacturer's 
protocol. PI (10 $\mu \mathrm{L}$ or $20 \mu \mathrm{L})$ was added to each tube and the cells gently re-suspended. Next, $400 \mu \mathrm{L}$ PBS were added to each tube. The number of stained cells was quantified using a flow cytometer (Becton Dickinson, New Jersey, USA).

Anti-influenza virus assay. Each compound was dissolved in DMSO at an initial concentration of $1000 \mu \mathrm{g} \mathrm{mL}{ }^{-1}$ and then successively diluted threefold to obtain 8 different concentrations as stock solutions. Madin-Darby canine kidney cells (MDCK) were seeded in 96-well trays and cultured at $37{ }^{\circ} \mathrm{C}$ in a humidified $\mathrm{CO}_{2}$ incubator (95\% air, $5 \% \mathrm{CO}_{2}$ ) for $24 \mathrm{~h}$. Then, the cells were infected with influenza A virus with $10^{-4}[316$ times the $50 \%$ tissue culture infective dose $\left.\left(\mathrm{TCID}_{50}\right)\right]$ and with influenza $B$ virus with $1 / 210^{-2}$ [158 times of the $50 \%$ tissue culture infective dose $\left.\left(\mathrm{TCID}_{50}\right)\right]$, respectively. All infected tissue culture plates (96 wells) were incubated at $37{ }^{\circ} \mathrm{C}$ for $2 \mathrm{~h}$, and then the medium was removed. Afterwards, $100 \mu \mathrm{L}$ aliquots of the solutions with different concentrations of each compound were added to the wells (one per well), and the plates were incubated again for $40 \mathrm{~h}$ at $37{ }^{\circ} \mathrm{C}$. Then, the inhibition of the virus-induced cytopathic effect (CPE) for each sample was recorded relative to the cell control and the virus control. ${ }^{46}$ The $50 \%$ cell-inhibitory concentration $\left(\mathrm{IC}_{50}\right)$ values of active compounds were calculated accordingly. The inhibitory potentials of the DDTD derivatives were comparable to those of the parent compound and the commercial drugs oseltamivir and RBV.

Cytotoxicity assay. MDCK cells were seeded in 96-well trays, each well 25000 cells and cultured at $37{ }^{\circ} \mathrm{C}$ in a humidified $\mathrm{CO}_{2}$ incubator $\left(95 \%\right.$ air, 5\% $\mathrm{CO}_{2}$ ) for $24 \mathrm{~h}$. Three times diluted compound was added cell monolayer and continued to cultivate for $48 \mathrm{~h}$. Then, the cytopathic effects were recorded. The 50\% cytotoxic concentration $\left(\mathrm{TC}_{50}\right)$ values of active compounds were calculated by the method of Reed and Muench.

\section{Acknowledgements}

This work was funded by the National Natural Science Foundation of China (NSFC) (Grant No. 21550110495) and Chinese Academy of Sciences President's International Fellowship Initiative (Grant No. 2016PT014).

\section{Notes and references}

1 M. Congreve, G. Chessari, D. Tisi and A. J. Woodhead, J. Med. Chem., 2008, 51, 3661-3680.

2 D. C. Rees, M. Congreve, C. W. Murray and R. Carr, Nat. Rev. Drug Discovery, 2004, 3, 660-672.

3 M. G. Siegel and M. Vieth, Drug Discovery Today, 2007, 12, 7179.

4 P. Bamborough and C.-w. Chung, MedChemComm, 2015, 6, 1587-1604.

5 J. Wang and T. Hou, J. Chem. Inf. Model., 2010, 50, 55-67. 6 S. Marino, D. Stachurska-Buczek, D. Huggins, B. Krywult, C. Sheehan, T. Nguyen, N. Choi, J. Parsons, P. Griffiths, I. James, A. Bray, J. White and R. Boyce, Molecules, 2004, 9, 405.
7 L. C. Blum and J.-L. Reymond, J. Am. Chem. Soc., 2009, 131, 8732-8733.

8 G. W. Bemis and M. A. Murcko, J. Med. Chem., 1996, 39, 2887-2893.

9 G. W. Bemis and M. A. Murcko, J. Med. Chem., 1999, 42, 5095-5099.

10 G. Schneider, M.-L. Lee, M. Stahl and P. Schneider, J. Comput.-Aided Mol. Des., 2000, 14, 487-494.

11 R. L. Siegel, K. D. Miller and A. Jemal, Ca-Cancer J. Clin., 2016, 66, 7-30.

12 C. Mugnaini, V. Pedani, D. Giunta, B. Sechi, M. Solinas, A. Casti, M. P. Castelli, G. Giorgi and F. Corelli, RSC Adv., 2014, 4, 1782-1793.

13 R. Romagnoli, P. G. Baraldi, C. Lopez-Cara, M. K. Salvador, D. Preti, M. A. Tabrizi, J. Balzarini, P. Nussbaumer, M. Bassetto, A. Brancale, X.-H. Fu, G. Yang, J. Li, S.-Z. Zhang, E. Hamel, R. Bortolozzi, G. Basso and G. Viola, Bioorg. Med. Chem., 2014, 22, 5097-5109.

14 D. Ye, Y. Zhang, F. Wang, M. Zheng, X. Zhang, X. Luo, X. Shen, H. Jiang and H. Liu, Bioorg. Med. Chem., 2010, 18, 1773-1782.

15 Y. Sun, J. Fan, Z. Zhu, X. Guo, T. Zhou, W. Duan and X. Shen, Eur. J. Pharmacol., 2015, 762, 202-213.

16 J. Thomas, A. Jejcic, P. Vervaeke, R. Romagnoli, S. Liekens, J. Balzarini and W. Dehaen, ChemMedChem, 2014, 9, 27442753.

17 A. Martorana, C. Gentile, U. Perricone, A. P. Piccionello, R. Bartolotta, A. Terenzi, A. Pace, F. Mingoia, A. M. Almerico and A. Lauria, Eur. J. Med. Chem., 2015, 90, 537-546.

18 A. Abu-Hashem, M. El-Shehry and F. Badria, Acta Pharm., 2010, 60, 311-323.

19 A. E. Rashad, A. H. Shamroukh, R. E. Abdel-Megeid, A. Mostafa, R. El-Shesheny, A. Kandeil, M. A. Ali and K. Banert, Eur. J. Med. Chem., 2010, 45, 5251-5257.

20 S. Massari, G. Nannetti, L. Goracci, L. Sancineto, G. Muratore, S. Sabatini, G. Manfroni, B. Mercorelli, V. Cecchetti, M. Facchini, G. Palù, G. Cruciani, A. Loregian and O. Tabarrini, J. Med. Chem., 2013, 56, 10118-10131.

21 S. Lepri, G. Nannetti, G. Muratore, G. Cruciani, R. Ruzziconi, B. Mercorelli, G. Palù, A. Loregian and L. Goracci, J. Med. Chem., 2014, 57, 4337-4350.

22 K. Bozorov, J.-Y. Zhao, B. Elmuradov, A. Pataer and H. A. Aisa, Eur. J. Med. Chem., 2015, 102, 552-573.

23 F. Han, S. Lin, P. Liu, X. Liu, J. Tao, X. Deng, C. Yi and H. Xu, ACS Med. Chem. Lett., 2015, 6, 434-438.

24 A. A. Gryshchenko, V. G. Bdzhola, A. O. Balanda, N. V. Briukhovetska, I. M. Kotey, A. G. Golub, T. P. Ruban, L. L. Lukash and S. M. Yarmoluk, Bioorg. Med. Chem., 2015, 23, 2287-2293.

25 S. Bugge, I. U. Moen, K.-O. Kragseth Sylte, E. Sundby and B. H. Hoff, Eur. J. Med. Chem., 2015, 94, 175-194.

26 F. Bysting, S. Bugge, E. Sundby and B. H. Hoff, RSC Adv., 2017, 7, 18569-18577.

27 K. Bozorov, H.-R. Ma, J.-Y. Zhao, H.-Q. Zhao, H. Chen, K. Bobakulov, X.-L. Xin, B. Elmuradov, K. Shakhidoyatov and H. A. Aisa, Eur. J. Med. Chem., 2014, 84, 739-745. 
28 A. M. Ginsberg, M. W. Laurenzi, D. J. Rouse, K. D. Whitney and M. K. Spigelman, Antimicrob. Agents Chemother., 2009, 53, 3720-3725.

29 R. Tiwari, G. C. Moraski, V. Krchňák, P. A. Miller, M. ColonMartinez, E. Herrero, A. G. Oliver and M. J. Miller, J. Am. Chem. Soc., 2013, 135, 3539-3549.

30 L. Zhou, G. Stewart, E. Rideau, N. J. Westwood and T. K. Smith, J. Med. Chem., 2013, 56, 796-806.

31 R. Mukkavilli, J. Pinjari, B. Patel, S. Sengottuvelan, S. Mondal, A. Gadekar, M. Verma, J. Patel, L. Pothuri, G. Chandrashekar, P. Koiram, T. Harisudhan, A. Moinuddin, D. Launay, N. Vachharajani, V. Ramanathan and D. Martin, Eur. J. Pharm. Sci., 2014, 65, 147-155.

32 A. M. Thompson, P. D. O'Connor, A. Blaser, V. Yardley, L. Maes, S. Gupta, D. Launay, D. Martin, S. G. Franzblau, B. Wan, Y. Wang, Z. Ma and W. A. Denny, J. Med. Chem., 2016, 59, 2530-2550.

33 A. C. Véras of Aguiar, R. O. of Moura, J. F. Bezerra Mendonça Junior, H. A. de Oliveira Rocha, R. B. Gomes Câmara and M. dos Santos Carvalho Schiavon, Biomed. Pharmacother., 2016, 84, 403-414.

34 K. Snegaroff, F. Lassagne, G. Bentabed-Ababsa, E. Nassar, S. C. S. Ely, H. Stephanie, E. Perspicace, A. Derdour and F. Mongin, Org. Biomol. Chem., 2009, 7, 4782-4788.

35 M. Bourgeaux, S. Vomscheid and W. G. Skene, Synth. Commun., 2007, 37, 3551-3558.
36 C. Isanbor and D. O'Hagan, J. Fluorine Chem., 2006, 127, 303319.

37 W. K. Hagmann, J. Med. Chem., 2008, 51, 4359-4369.

38 E. Asadi, M. Abdouss, R. M. Leblanc, N. Ezzati, J. N. Wilson and S. Azodi-Deilami, RSC Adv., 2016, 6, 37308-37318.

39 E. A. K. Nivethaa, S. Dhanavel, V. Narayanan, C. A. Vasu and A. Stephen, $R S C$ Adv., 2015, 5, 1024-1032.

40 M. Dauria and G. Mauriello, Photochem. Photobiol., 1994, 60, 542-545.

41 R. R. Tykwinski, A. Hilger, F. Diederich, H. P. Lüthi, P. Seiler, V. Gramlich, J.-P. Gisselbrecht, C. Boudon and M. Gross, Helv. Chim. Acta, 2000, 83, 1484-1508.

42 P. Thomson, M. A. Naylor, S. A. Everett, M. R. L. Stratford, G. Lewis, S. Hill, K. B. Patel, P. Wardman and P. D. Davis, Mol. Cancer Ther., 2006, 5, 2886-2894.

43 J. Weinstock, J. Wu, P. Cao, W. D. Kingsbury, J. L. McDermott, M. P. Kodrasov, D. M. McKelvey, K. G. Suresh Kumar, S. J. Goldenberg, M. R. Mattern and B. Nicholson, ACS Med. Chem. Lett., 2012, 3, 789-792.

44 R. W. Pero, A. Olsson, A. Amiri and D. Chaplin, Cancer Detect. Prev., 1998, 22, 225-236.

45 Y. Zhang, L. Jin, H. Xiang, J. Wu, P. Wang, D. Hu, W. Xue and S. Yang, Eur. J. Med. Chem., 2013, 66, 335-344.

46 J. Zhao and H. A. Aisa, Bioorg. Med. Chem. Lett., 2012, 22, 2321-2325. 